Mikhail Feldman · John McCuan

\title{
Constructing convex solutions via Perron's method
}

Received: 18 December 2003 / Accepted: 5 July 2005 / Published online: 16 April 2007 (c) 2007 The Authors ${ }^{1}$

\begin{abstract}
In this paper we construct convex solutions for certain elliptic boundary value problems via Perron's method. The solutions constructed are weak solutions in the viscosity sense, and our construction follows work of Ishii (Duke Math. J., 55 (2) 369-384, 1987). The same general approach appears in work of Andrews and Feldman (J. Differential Equations, 182 (2) 298-343, 2002) in which they show existence for a weak nonlocal parabolic flow of convex curves. The time independent special case of their work leads to a one dimensional elliptic result which we extend to two dimensions. Similar results are required to extend their theory of nonlocal geometric flows to surfaces.

The two dimensional case is essentially different from the one dimensional case and involves a regularity result (cf. Theorem 3.1), which has independent interest. Roughly speaking, given an arbitrary convex function (which is not smooth) supported at one point by a smooth function of prescribed Hessian (which is not convex), one must construct a third function that is both convex and smooth and appropriately approximates both of the given functions.
\end{abstract}

Keywords Viscosity solutions · Elliptic partial differential equations · Perron procedure $\cdot$ Convexity $\cdot$ Regularity $\cdot$ Fully nonlinear $\cdot$ Monge-Ampere

Mathematics Subject Classification (2000) 35J60 · 53A05 · 52A15 - 26B05

The Managing Editor states that the long delay was due to an oversight on the part of the journal.

M. Feldman

Department of Mathematics University of Wisconsin, 709 Van Vleck Hall, 480 Lincoln Drive Madison, WI 53706

E-mail: feldman@math.wisc.edu

J. McCuan ( $\varangle)$

Georgia Institute of Tecnology, Atlanta, GA 30332-0160

E-mail: mccuan@math.gatech.edu

${ }^{1}$ This is a "Springer Open Choice" article. Unrestricted non-commercial use, distribution, and reproduction in any medium is permitted, provided the original author and source are credited. 


\section{Introduction}

There has been considerable interest recently in spatially convex viscosity solutions for elliptic and parabolic pde. We mention specifically the paper [1] of Alvarez, Lazry and Lions which gives a method for showing convexity of solutions once the existence has been established. In the absence of apriori existence (when there is no comparison principle, for example) or when one has a family of interrelated equations $L u=f=u_{t}$, it is useful to establish the existence of convex solutions directly. This was the point of view taken in [2] and the point of view we take in this paper.

Generally, we consider a convex domain $\Omega \subset \mathbb{R}^{n}$ and an elliptic equation of the form

$$
F\left(x, u, D u, D^{2} u\right)=0
$$

where the operator $F: \bar{\Omega} \times \mathbb{R} \times \mathbb{R}^{n} \times \mathscr{S}^{n \times n} \rightarrow \mathbb{R}$ is assumed to be continuous. Ellipticity, in this context, means that $F(x, z, p, A) \geq F(x, z, p, B)$ whenever $A$ and $B$ are symmetric matrices and $A-B$ is nonnegative semidefinite (i.e., $A \geq B$ ). We will also assume the A-L-L condition from [1] that for fixed $p$ the map

$$
(x, z, A) \mapsto F\left(x, z, p, A^{-1}\right) \text { is convex on } \Omega \times \mathbb{R} \times \mathscr{S}_{+}^{n \times n},
$$

where $\mathscr{S}_{+}^{n \times n}$ denotes the positive definite symmetric matrices.

A subsolution (following [3]) is a function $u \in C^{0}(\Omega)$ for which

$$
F\left(x, u(x), D u(x), D^{2} u(x)\right) \geq 0
$$

weakly. That is to say, whenever, $x \in \Omega$ and $\phi \geq u$ is a smooth function with $\phi(x)=u(x)$, then

$$
F\left(x, u(x), D \phi(x), D^{2} \phi(x)\right) \geq 0 .
$$

Supersolutions are defined similarly, and a solution is a continuous function which is both a subsolution and a supersolution.

The situation in which a smooth function $\phi$ has graph touching the graph of a continuous function $u$ from one side will arise many times in the discussion below. We use the following notation:

If $\phi \leq u$ is smooth with $\phi(x)=u(x)$, we write $\phi \in \Xi^{-} u(x)$.

Writing $\phi \in \Xi^{+} u(x)$ indicates the similar situation in which $\phi \geq u$.

If $(p, A) \in \mathbb{R}^{n} \times \mathscr{S}^{n \times n}$ and there is some $\phi \in \Xi^{ \pm} u(x)$ with $(p, A)=$ $\left(D \phi(x), D^{2} \phi(x)\right)$, we write $(p, A) \in J^{ \pm} u(x)$, the second order super/sub jet of $u$ at $x$.

We will also consider the closure of jets as follows:

We write $(p, A) \in \operatorname{clos}\left[J^{ \pm} u(x)\right]$ if there are sequences $x_{j} \rightarrow x$ and $\left(p_{j}, A_{j}\right) \rightarrow$ $(p, A)$ with $\left(p_{j}, A_{j}\right) \in J^{ \pm} u\left(x_{j}\right)$.

A function $u$ satisfies state constraints on $\partial \Omega$ if for every $x \in \partial \Omega$ and $(p, A) \in$ $J^{-} u(x)$, one has

$$
F(x, u(x), p, A) \leq 0 .
$$

Finally, $u$ satisfies strong state constraints on $\partial \Omega$ if either 
(a) the subjet of $u$ at each point in $\partial \Omega$ is empty, or

(b) $u$ satisfies state constraints and $F$ is totally degenerate on $\partial \Omega$, i.e., $F=F(x, u, D u)$.

We remark that (a) holds if the outward normal derivative of $u$ (exists and) is $+\infty$ or if $u$ satisfies state constraints and $F$ is uniformly elliptic on $\partial \Omega$, i.e., $F(x, z, p, A+B) \geq F(x, z, p, A)+\lambda \operatorname{tr} B$ for $B \geq 0$. To see that the latter conditions imply (a), suppose, without loss of generality, that $x=0 \in \partial \Omega$ and $\Omega \subset\left\{x_{1} \leq 0\right\}$. If $\phi \in \Xi^{-} u(0)$, then set $p=D \phi(0)$ and $\tilde{\phi}=u(0)+p \cdot x+x_{1}+M x_{1}^{2}$. It follows that $\tilde{\phi} \in \Xi^{-} u(0)$ for any $M>0$ and

$$
F\left(0, u(0), p+e_{1}, 2 M e_{1} e_{1}^{\mathrm{t}}\right) \geq F\left(0, u(0), p+e_{1}, 0\right)+2 \lambda M .
$$

This last assertion violates (1.3) when $M$ is sufficiently large. A similar reasoning applies without uniform ellipticity for equations of Monge-Ampere type $\operatorname{det} D^{2} u=$ $\phi(D u)$, if for each $x \in \partial \Omega$, there is a ball $B$ with $\Omega \subset B$ and $x \in \partial B$. In this case, we take $\tilde{\phi}=u(0)+p \cdot x+x_{1}+\epsilon\left(x_{2}^{2}+\cdots+x_{n}^{2}\right)+M x_{1}^{2}$ and obtain a contradiction for $\epsilon$ small and $M$ large.

We prove the following result for $n \leq 2$.

\section{Theorem 1.1 If the equation (1.1) admits}

(i) a supersolution $v_{0} \in C^{0}(\bar{\Omega})$ satisfying strong state constraints on $\partial \Omega$, and

(ii) a convex subsolution $u_{0} \in C^{0}(\bar{\Omega})$ with $u_{0}=v_{0}$ on $\partial \Omega$,

then there exists a convex solution of (1.1) on $\Omega$.

It should be noted that the solution given by Theorem 1.1 is not obtained using a comparison principle as in [1]. Therefore, the theorem applies to a broader range of equations and, in particular, solutions may not be unique.

We note that any quasilinear elliptic equation $\operatorname{tr}\left[a(D u) D^{2} u\right]=\phi(D u)$ satisfies (1.2) as do Monge-Ampere equations of the form $\operatorname{det} D^{2} u=\phi(D u)$.

\section{Perron's Procedure}

In the classical Perron procedure [4], one sets

$$
u_{1}(x)=\sup \left\{u(x): u \in C^{0}(\bar{\Omega}) \text { is a subsolution with } u_{\left.\right|_{\partial \Omega}} \leq g\right\}
$$

for some given boundary values $g$ and argues that $u=u_{1}$ is a solution. One makes the same definition in the analogous argument for weak solutions in the viscosity sense [5]. Our assumption allows us to restrict the supremum to convex subsolutions:

$$
u_{1}(x)=\sup \left\{u(x): u \in C^{0}(\bar{\Omega}) \text { is a convex subsolution with } u \leq v_{0}\right\} .
$$

In all three cases, it is fairly straightforward to see that $u=u_{1}$ is a subsolution. In our case, furthermore, it is clear that $u=u_{1}$ is convex. Thus, it only remains to show that $u=u_{1}$ is a supersolution. The remainder of the paper is devoted to proving this point.

If one sets convexity aside, then the proof is completed via some form of the following fundamental lemma. 
Lemma 2.1 (Ishii's Lemma) If $u$ is a subsolution, and $\phi \in \Xi^{-} u\left(x_{0}\right)$ with

A. $F\left(x_{0}, u\left(x_{0}\right), D \phi\left(x_{0}\right), D^{2} \phi\left(x_{0}\right)\right)>0$, and

B. $\phi(x)<u(x), x \neq x_{0}$,

then for $\delta$ small, $\hat{u}=\max \{u, \phi+\delta\}$ is a subsolution with $\hat{u}_{\left.\right|_{\partial \Omega}} \leq g$.

In fact, the assumption that $u=u_{1}$ is not a supersolution is exactly equivalent to the existence of a smooth function $\phi$ satisfying all conditions of the lemma except the strict inequality $\mathbf{B}: \phi(x)<u(x)$ for $x \neq x_{0}$. Replacing $\phi$ with $\phi-\epsilon\left|x-x_{0}\right|^{2}$, it is clear from continuity that all the requirements of Ishii's lemma can be assumed. The conclusion of the lemma, then, is a direct contradiction of the definition of $u_{1}$ given in (2.1).

In the convex case we are considering, there is no way to know that $\hat{u}=$ $\max \{u, \phi+\delta\}$ is convex unless some care is taken in obtaining the smooth supporting function $\phi$. In fact, it is not generally possible to choose such a function in $\Xi^{-} u\left(x_{0}\right)$. Nevertheless, we have recourse to the following somewhat technical generalization of Lemma 2.1.

Lemma 2.2 (New Fundamental Lemma) If $u \leq v_{0}$ is a convex subsolution, $\left(p_{0}, A_{0}\right) \in \mathbb{R}^{n} \times \mathscr{S}^{n \times n}$ with

A. $F\left(x_{0}, u\left(x_{0}\right), p_{0}, A_{0}\right)>0$,

for any $\delta_{0}, \epsilon_{0}>0$ there is a smooth convex function $\psi$ defined on a subset $U \subset$ $B_{\delta_{0}}\left(x_{0}\right)$ such that

B. $\max _{x \in U}[\psi(x)-u(x)]>0$,

C. $\left|\psi-\psi_{0}\right|_{C^{1}(U)}<\epsilon_{0}$ where $\psi_{0}(x)=u\left(x_{0}\right)+p_{0} \cdot\left(x-x_{0}\right)$,

$$
D^{2} \psi(x) \geq A_{0}-\epsilon_{0} I \quad \text { for } x \in U, \text { and }
$$

D. $\psi_{\left.\right|_{\partial U}}<u_{\left.\right|_{\partial U}}$,

then for $\delta_{0}, \epsilon_{0}$ small, $\hat{u}=\max \{u, \psi\}$ is a convex subsolution and $\hat{u} \leq v_{0}$.

Remark 2.1 If the domain $U$ is not convex, then we require $\psi$ to be defined (and convex) on a convex domain $W \supset U$.

Remark 2.2 In order to verify condition $\mathbf{B}$ it is enough to show $\max _{x \in U}[\psi(x)-$ $u(x)] \geq 0$. (Replacing $\psi$ by $\psi+\delta_{1}$ for some small $\delta_{1}$, one sees that all hypotheses hold.) Similarly, one can relax the strict inequality in D. (Replacing $\psi$ by $\psi-\delta_{1}$ one gets the strict inequality with all other conditions still satisfied.)

We refer to the function $\psi$ appearing in the lemma as a direct modifying function. Notice that one may subtract an appropriate constant from $\psi$ to obtain a function in $\Xi^{-} u\left(\tilde{x}_{0}\right)$ for some point or points $\tilde{x}_{0} \in \Omega$, but the lemma gives little control on the location or number of such points, save that they are close to $x_{0}$.

The proof of Lemma 2.2 is relatively straightforward, and we conclude this section with that. The construction of a modifying function $\psi$ satisfying the hypotheses of the lemma is fairly involved and will be given in the next section. It should be noted, however, that if such a function exists, then in light of condition $\mathbf{B}$, the modification $\hat{u}$ is nontrivial and gives a contradiction just like Ishii's Lemma. 
Proof (of Lemma 2.2.) In light of $\mathbf{A}$, when $\epsilon_{0}$ is small enough, we have

$$
F\left(x_{0}, u\left(x_{0}\right), p_{0}, A_{0}-\epsilon_{0} I\right)>0 .
$$

By the continuity of $F$, there is some $\delta_{1}$ for which

$$
F\left(x, z, p, A_{0}-\epsilon_{0} I\right)>0 \quad \text { whenever } \quad\left|(x, z, p)-\left(x_{0}, u\left(x_{0}\right), p_{0}\right)\right|<\delta_{1} .
$$

By the smoothness of $\psi_{0}$ there is some $\delta_{2}>0$ for which

$$
\left|\left(x, \psi_{0}(x), D \psi_{0}(x)\right)-\left(x_{0}, u\left(x_{0}\right), p_{0}\right)\right|<\delta_{1} / 10 \quad \text { whenever } \quad\left|x-x_{0}\right|<\delta_{2} .
$$

Let

$$
\delta_{0}<\min \left\{\frac{\delta_{1}}{6}, \delta_{2}\right\} \quad \text { and } \quad \epsilon_{0}<\frac{\delta_{1}}{4} .
$$

Because $U \subset B_{\delta_{0}}\left(x_{0}\right)$ and condition $\mathbf{D}$ holds, we see that $\hat{U}=\{x: \hat{u}(x)>u(x)\} \subset$ $B_{\delta_{0}}\left(x_{0}\right)$.

If $x \in \Omega \backslash \hat{U}$, then $\hat{u}(x)=u(x)$, and any smooth function $\eta \in \Xi^{+} \hat{u}(x)$ has also $\eta \in \Xi^{+} u(x)$. Since $u$ is a subsolution, we have

$$
F\left(x, \hat{u}(x), D \eta(x), D^{2} \eta(x)\right) \geq 0 .
$$

If, on the other hand, $x \in \hat{U}$, then $\hat{u}(x)=\psi(x)$, and any $\eta \in \Xi^{+} \hat{u}(x)$ must satisfy $D \eta(x)=D \psi(x)$ and $D^{2} \eta(x) \geq D^{2} \psi(x) \geq A_{0}-\epsilon_{0} I$. The last inequality is from C. By ellipticity therefore,

$$
F\left(x, \hat{u}(x), D \eta(x), D^{2} \eta(x)\right) \geq F\left(x, \hat{u}(x), D \psi(x), A_{0}-\epsilon_{0} I\right) .
$$

Note, however, that

$$
\left|(x, \hat{u}(x), D \psi(x))-\left(x_{0}, u\left(x_{0}\right), p_{0}\right)\right| \leq\left|x-x_{0}\right|+\left|\psi(x)-u\left(x_{0}\right)\right|+\left|D \psi(x)-p_{0}\right| .
$$

We consider each term on the right separately. Since $x \in \hat{U} \subset B_{\delta_{0}}\left(x_{0}\right)$, we have by the choice (2.5) of $\delta_{0}$,

$$
\left|x-x_{0}\right|<\min \left\{\frac{\delta_{1}}{6}, \delta_{2}\right\} .
$$

Estimating the second term,

$$
\begin{aligned}
\left|\psi(x)-u\left(x_{0}\right)\right| & \leq\left|\psi(x)-\psi_{0}(x)\right|+\left|\psi_{0}(x)-u\left(x_{0}\right)\right| \\
& <\epsilon_{0}+\delta_{1} / 6 .
\end{aligned}
$$

The second inequality uses $\mathbf{C}$ and (2.4) along with the fact (2.7) that $\left|x-x_{0}\right|<\delta_{2}$.

The third term is similar to the second:

$$
\begin{aligned}
\left|D \psi(x)-p_{0}\right| & \leq\left|D \psi(x)-D \psi_{0}(x)\right|+\left|D \psi_{0}(x)-p_{0}\right| \\
& <\epsilon_{0}+\delta_{1} / 6 .
\end{aligned}
$$


Summing these estimates and using the choices (2.5) of $\delta_{0}$ and $\epsilon_{0}$, we obtain

$$
\begin{aligned}
\left|(x, \hat{u}(x), D \psi(x))-\left(x_{0}, u\left(x_{0}\right), p_{0}\right)\right| & \leq \frac{\delta_{1}}{2}+2 \epsilon_{0} \\
& <\delta_{1} .
\end{aligned}
$$

In light of (2.3) and (2.6), we have

$$
\begin{aligned}
F\left(x, \hat{u}(x), D \eta(x), D^{2} \eta(x)\right) & \geq F\left(x, \hat{u}(x), D \psi(x), A_{0}-\epsilon_{0} I\right) \\
& >0 .
\end{aligned}
$$

Thus, $\hat{u}$ is a subsolution.

The function $\hat{u}$, being the maximum of two convex functions, is also convex.

Furthermore, since $v_{0}$ is a subsolution, it cannot be the case that $u\left(x_{0}\right)=v_{0}\left(x_{0}\right)$. By continuity, it follows that $\hat{u} \leq v_{0}$ for $\delta$ small.

\section{Modification}

We now embark on the proof of the main result, which is by contradiction. Accordingly, we make the standing assumption that

$$
u=u_{1}(x)=\sup \left\{u(x): u \in C^{0}(\bar{\Omega}) \text { is a convex subsolution with } u \leq v_{0}\right\}
$$

is not a supersolution. The following result, which holds in the convex case, is recorded in [1, Lemma 3].

Lemma 3.1 There is some $x_{0} \in \Omega$ and some $\phi \in \Xi^{-} u\left(x_{0}\right)$ for which

$$
F\left(x_{0}, u\left(x_{0}\right), D \phi\left(x_{0}\right), D^{2} \phi\left(x_{0}\right)\right)>0
$$

and

$$
D^{2} \phi\left(x_{0}\right) \geq 0
$$

Remark 3.1 The inequality on the operator is a direct consequence of our assumption that $u$ is not a supersolution. The content of the lemma is (3.3).

Next, we distinguish two possibilities. The first is that

$$
X_{0}=\left(x_{0}, u\left(x_{0}\right)\right) \text { is an extreme point of } \mathscr{G}=\operatorname{graph}(u),
$$

by which we mean that $X_{0}$ belongs to the interior of no segment on $\mathscr{G}$ (symbolically, if $(1-\lambda) X_{1}+\lambda X_{2}=X_{0}$ for $X_{1}, X_{2} \in \mathscr{G}$ and $\lambda \in[0,1]$, then $X_{1}=X_{0}$ or $X_{2}=X_{0}$ ). The alternative is, of course, that

$$
X_{0}=\left(x_{0}, u\left(x_{0}\right)\right) \text { is not an extreme point of } \mathscr{G} .
$$

Since possibility (3.4) will be subsumed under more general considerations taken up below, we focus for the moment on (3.5). 
In this case, there are $x_{1}, \ldots, x_{k} \in \bar{\Omega}$ and $\lambda_{1}, \ldots, \lambda_{k} \in(0,1)$ with $\sum \lambda_{i}=1$ such that $X_{i}=\left(x_{i}, u\left(x_{i}\right)\right)$ is an extreme point for each $i$,

$$
x_{0}=\sum_{i=1}^{k} \lambda_{i} x_{i}
$$

and

$$
u\left(x_{0}\right)=\sum_{i=1}^{k} \lambda_{i} u\left(x_{i}\right) .
$$

Furthermore, as described in [2, Proposition 28], there exist $A_{i} \geq 0$ with $\left(p, A_{i}\right) \in$ $\operatorname{clos}\left[J^{-} u\left(x_{i}\right)\right]$ where $p=D \phi\left(x_{0}\right)$. A key estimate from [1] then asserts that for any $\epsilon>0$, the $A_{i}$ can be chosen to satisfy

$$
D^{2} \phi\left(x_{0}\right)-\epsilon\left[D^{2} \phi\left(x_{0}\right)\right]^{2} \leq \lim _{\delta \rightarrow 0}\left[\sum_{i=1}^{k} \lambda_{i}\left(A_{i}+\delta I\right)^{-1}\right]^{-1} .
$$

We claim that at one of the points $x_{i}$ we have

$$
F\left(x_{i}, u\left(x_{i}\right), p, A_{i}\right)>0 .
$$

Were the reverse inequality to hold at all points, then

$$
\begin{aligned}
F\left(x_{0}, u\left(x_{0}\right), D \phi\left(x_{0}\right),\right. & \left.D^{2} \phi\left(x_{0}\right)-\epsilon\left[D^{2} \phi\left(x_{0}\right)\right]^{2}\right) \\
& \leq F\left(\sum \lambda_{i} x_{i}, \sum \lambda_{i} u\left(x_{i}\right), p, \lim _{\delta \rightarrow 0}\left[\sum \lambda_{i}\left(A_{i}+\delta I\right)^{-1}\right]^{-1}\right) \\
& \leq \lim _{\delta \rightarrow 0} \sum \lambda_{i} F\left(x_{i}, u\left(x_{i}\right), p, A_{i}+\delta I\right) \\
& =\sum \lambda_{i} F\left(x_{i}, u\left(x_{i}\right), p, A_{i}\right) \\
& \leq 0 .
\end{aligned}
$$

The first inequality uses (3.6) and ellipticity; the second follows from the convexity property (1.2) of $F$; the last inequality uses the negation of (3.7). Letting $\epsilon$ tend to 0 , we obtain a contradiction to (3.2).

The particular point $x_{i}$ for which (3.7) holds, owing to the strong state constraints, must be an interior point of $\Omega$. We now focus our attention on what happens near $x_{i}$ and, accordingly, drop the $i$-subscript, rename the point $x_{0}$, and write $p=p_{0}, A_{i}=A_{0}$. Condition (3.7) becomes

$$
F\left(x_{0}, u\left(x_{0}\right), p_{0}, A_{0}\right)>0 .
$$

It is important to note that we no longer have a smooth function $\phi \in \Xi^{-} u\left(x_{0}\right)$. On the other hand, the situation in which we find ourselves covers possibility (3.4) as suggested above. We are now in a position to formulate our main modification result which handles both cases.

Theorem 3.1 Let $u$ be a convex function defined in a neighborhood of $x_{0} \in \mathbb{R}^{2}$. Assume that $A_{0} \geq 0$ and $\left(p_{0}, A_{0}\right) \in \operatorname{clos}\left[J^{-} u\left(x_{0}\right)\right]$. If $X_{0}=\left(x_{0}, u\left(x_{0}\right)\right)$ is an extreme point for $\mathscr{G}=\operatorname{graph}(u)$, then for any $\delta_{0}, \epsilon_{0}>0$, there exists a smooth convex function $\psi$ defined in a domain $V \subset B_{\delta_{0}}\left(x_{0}\right)$ such that 
B. $\max _{x \in V}[\psi(x)-u(x)]>0$,

$\mathbf{C}_{1}$. for every $x \in \hat{V}=\{x \in V: \psi(x)>u(x)\}$,

$$
\begin{gathered}
\left|\psi(x)-u\left(x_{0}\right)\right|+\left|D \psi(x)-p_{0}\right|<\epsilon_{0}, \\
D^{2} \psi(x) \geq A_{0}-\epsilon_{0} I, \quad \text { and }
\end{gathered}
$$

$\mathbf{D}_{1 .} \psi_{\left.\right|_{\partial \hat{V}}} \leq u_{\left.\right|_{\partial \hat{V}}}$.

If we take $\left(p_{0}, A_{0}\right)$ in Theorem 3.1 to be the pair in $\operatorname{clos}\left[J^{-} u\left(x_{0}\right)\right]$ satisfying (3.8), then all the hypotheses of Lemma 2.2 may be verified as follows. Let $\delta_{0}, \epsilon_{0}$ be given from Lemma 2.2. Take $\tilde{\epsilon}_{0}, \tilde{\delta}_{0}<1$ from Theorem 3.1 such that

$$
\tilde{\epsilon}_{0} \leq \epsilon_{0} / 4
$$

and

$$
\tilde{\delta}_{0} \leq \min \left\{\delta_{0}, \epsilon_{0} /\left(\left|p_{0}\right|+1\right)\right\} / 2 .
$$

Taking $\psi$ to be the function given by Theorem 3.1 and $U=\hat{V}$, we see that $\mathbf{B}$ follows from $\mathbf{B}_{1}$. Next, we see that

$$
\begin{aligned}
\left|\psi-\psi_{0}\right|_{C^{1}(U)} & =\sup _{x \in \hat{V}}\left|\psi(x)-\psi_{0}(x)\right|+\sup _{x \in \hat{V}}\left|D \psi(x)-p_{0}\right| \\
& \leq \sup _{x \in \hat{V}}\left|\psi(x)-u\left(x_{0}\right)\right|+\sup _{x \in \hat{V}}\left|D \psi(x)-p_{0}\right|+\sup _{x \in \hat{V}}\left|p_{0}\right|\left|x-x_{0}\right| \\
& \leq 2 \tilde{\epsilon}_{0}+\left|p_{0}\right| \tilde{\delta}_{0} \\
& <\epsilon_{0} .
\end{aligned}
$$

This is the first requirement of $\mathbf{C}$; the second requirement is given verbatim in Theorem 3.1. Finally, condition $\mathbf{D}_{1}$ is exactly the weakened form of condition $\mathbf{D}$, which is enough by Remark 2.2.

Proof (of Theorem 3.1) By definition there is a sequence $x_{j} \rightarrow x_{0}$ with corresponding $\phi_{j} \in \Xi^{-} u\left(x_{j}\right)$ and $\left(u\left(x_{j}\right), D \phi_{j}\left(x_{j}\right), D^{2} \phi_{i}\left(x_{i}\right)\right) \rightarrow\left(u\left(x_{0}\right), p_{0}, A_{0}\right)$. While we know $A_{0} \geq 0$, we do not know $D^{2} \phi_{j}\left(x_{j}\right) \geq 0$. Our first task will be to obtain an alternative sequence of points at which to make our modification. In the process, we will give up the convergence of the Hessian.

Lemma 3.2 For any $\alpha_{0}>0$, there is some $\tilde{x} \in \Omega$ and $\tilde{\phi} \in \Xi^{-} u(\tilde{x})$ satisfying

(i) $D^{2} \tilde{\phi}(\tilde{x}) \geq 0$

(ii) $\left|\tilde{x}-x_{0}\right|+\left|D \tilde{\phi}(\tilde{x})-p_{0}\right|<\alpha_{0}$,

(iii) $D^{2} \tilde{\phi}(\tilde{x}) \geq A_{0}-\alpha_{0} I$.

We use Jensen's distance convolution to prove Lemma 3.2. The following proposition records the relevant properties $[3,6,7]$. Recall that $\mathscr{G}=\operatorname{graph}(u)$.

Proposition 3.1 For $\epsilon>0$ set

$$
\mathscr{G}_{\epsilon}=\{(x, z) \in \Omega \times(-\infty, u(x)]: \operatorname{dist}[(x, z), \mathscr{G}]=\epsilon\} .
$$

a. $\mathscr{G}_{\epsilon}$ is the graph of a function $\check{u}=\check{u}_{\epsilon}$ on $\Omega$. Clearly, $\check{u}$ converges uniformly on compact subsets to u as $\epsilon \rightarrow 0$. 
b. The function $\check{u}$ is convex and, consequently, twice differentiable almost everywhere.

c. $\mathscr{G}_{\epsilon}$ is locally touchable, i.e., for any $\check{x} \in \Omega, r, \delta>0$, and $\check{\phi} \in \Xi^{-} \check{u}(\check{x})$ with $\check{\phi}(x)<\check{u}(x)$ for $x \neq \check{x}$, the set

$$
\left\{\xi \in B_{r}(\check{x}): \check{\phi}+\check{\ell} \in \Xi^{-} \check{u}(\xi) \text { for some } \check{\ell}=\check{p} \cdot(x-\xi)+\check{z} \text { with } \check{p} \in B_{\delta}(0)\right\}
$$

has positive measure. (Note that $\check{z}=\check{u}(\xi)-\breve{\phi}(\xi)$ is prescribed when $\xi$ is in the set).

Proof (of Lemma 3.2) There is some $\alpha_{1}>0$ for which

$$
\left|A-A_{0}\right|<\alpha_{1} \quad \Longrightarrow \quad A \geq-\frac{\alpha_{0}}{2} I .
$$

By taking $j$ large enough, we have $\phi_{j} \in \Xi^{-} u\left(x_{j}\right)$ with

$$
\left|x_{j}-x_{0}\right|+\left|D \phi_{j}\left(x_{j}\right)-p_{0}\right|+\left|D^{2} \phi_{j}\left(x_{j}\right)-A_{0}\right|<\min \left\{\frac{\alpha_{0}}{3}, \frac{\alpha_{1}}{2}\right\} \text {. }
$$

Replacing $\phi_{j}(x)$ with $\phi_{j}(x)-\left|x-x_{j}\right|^{2} / j$ if necessary, we may also assume

$$
\phi_{j}(x)<u(x) \text { for } x \neq x_{j} .
$$

If follows from (3.11) and Proposition 3.1a that we may take $\epsilon$ small enough and find a point $\check{x} \in \Omega$ and a smooth function $\breve{\phi} \in \Xi^{-} \check{u}(\check{x})$ such that

$$
\left|\check{x}-x_{j}\right|+\left|D \check{\phi}(\check{x})-D \phi_{j}\left(x_{j}\right)\right|+\left|D^{2} \check{\phi}(\check{x})-D^{2} \phi_{j}\left(x_{j}\right)\right|+\epsilon<\min \left\{\frac{\alpha_{0}}{3}, \frac{\alpha_{1}}{2}\right\} .
$$

In fact, since $\check{u}$ converges uniformly to $u$ and $u(x)>\phi_{j}(x)$ for $x \neq x_{j}$, we have for fixed $r$,

$$
C=\left\{x: \phi_{j}(x) \geq \check{u}(x)\right\} \subset B_{r}\left(x_{j}\right)
$$

if $\epsilon$ is small enough; see Figure 1. Taking some $\check{x} \in C$ such that $m=\phi_{j}(\check{x})-\check{u}(\check{x})=$ $\max \left(\phi_{j}-\check{u}\right)$, we set $\breve{\phi}=\phi_{j}-m$, and (3.12) follows by continuity.

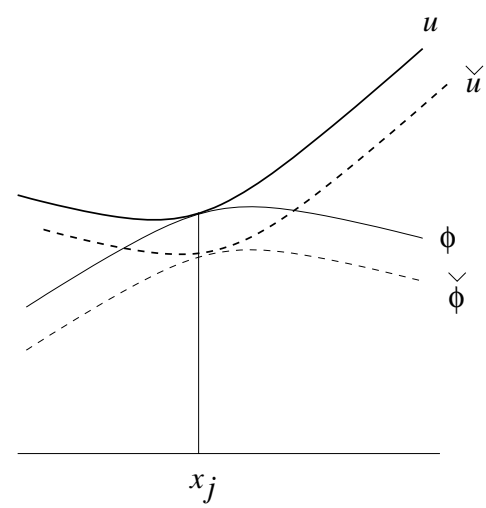

Fig. 1 The subjet of Jensen's Convolution 
Replacing $\check{\phi}(x)$ with $\breve{\phi}(x)-|x-\check{x}|^{2} / k$ for $k$ large enough, we may also assume $\check{\phi}(x)<\check{u}(x)$ for $x \neq \check{x}$. Therefore, Proposition 3.1c applies. Coupling this with Proposition $3.1 \mathrm{~b}$, we obtain a point of twice differentiability $\hat{\xi}$ such that

$$
\check{\psi}(x)=\check{\phi}(x)+\check{p} \cdot(x-\check{\xi})+\check{u}(\check{\xi})-\check{\phi}(\check{\xi})
$$

satisfies $\check{\psi} \in \Xi^{-} \breve{u}(\breve{\xi})$. Furthermore, we may take the $r$ and $\delta$ of Proposition 3.1 so small that

$$
|\check{\xi}-\check{x}|+|D \check{\psi}(\check{\xi})-D \check{\phi}(\check{x})|<\frac{\alpha_{0}}{3}
$$

and

$$
D^{2} \breve{\psi}(\check{\xi}) \geq D^{2} \check{\phi}(\check{x})-\frac{\alpha_{0}}{2} I .
$$

Since $\breve{\xi}$ is a point of twice differentiability of $\breve{u}$, there is a smooth function $\tilde{\psi} \in \Xi^{-} \check{u}(\breve{\xi})$ with

$$
D^{2} \tilde{\psi}(\breve{\xi})=D^{2} \breve{u}(\check{\xi}) \geq D^{2} \check{\psi}(\check{\xi})
$$

see [9].

Let $\check{X}=(\breve{\xi}, \breve{u}(\breve{\xi}))$. It follows from the convexity of $u$ that there is a unique $\tilde{X}=(\tilde{x}, u(\tilde{x})) \in \mathscr{G}$ with

$$
|\tilde{X}-\check{X}|=\epsilon ;
$$

see Figure 2. Again referring to Figure 2, we set

$$
\tilde{\phi}(x)=\tilde{\psi}(x-\tilde{x}+\check{\xi})+u(\tilde{x})-\check{u}(\check{\xi}) .
$$

We claim that $\tilde{x}$ and $\tilde{\phi}$ satisfy the assertions of Lemma 3.2.

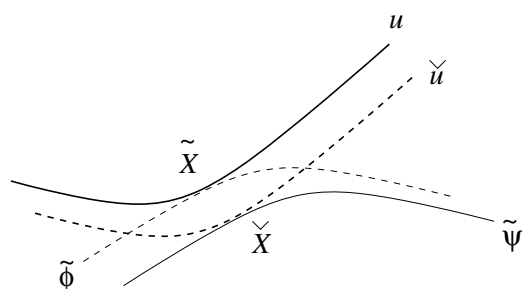

Fig. 2 A twice differentiable point on Jensen's Convolution

We first verify that $\tilde{\phi} \in \Xi^{-} u(\tilde{x})$. Equality at $\tilde{x}$ is clear since $\tilde{\psi} \in \Xi^{-} \breve{u}(\check{\xi})$. If we assume $\tilde{\phi}(x)>u(x)$, then

$$
\begin{aligned}
u(x)-\check{u}(x-\tilde{x}+\check{\xi}) & <\tilde{\phi}(x)-\check{u}(x-\tilde{x}+\check{\xi}) \\
& =\tilde{\psi}(x-\tilde{x}+\check{\xi})-\check{u}(x-\tilde{x}+\check{\xi})+u(\tilde{x})-\check{u}(\check{\xi}) \\
& \leq u(\tilde{x})-\check{u}(\check{\xi}) .
\end{aligned}
$$


Therefore,

$$
\begin{aligned}
\operatorname{dist}[(x-\tilde{x}+\check{\xi}), \mathscr{G}] & \leq\left[(\tilde{x}-\check{\xi})^{2}+(u(x)-\check{u}(x-\tilde{x}+\check{\xi}))^{2}\right]^{1 / 2} \\
& <\left[(\tilde{x}-\check{\xi})^{2}+(u(\tilde{x})-\check{u}(\check{\xi}))^{2}\right]^{1 / 2} \\
& =\epsilon
\end{aligned}
$$

by (3.16), which is a contradiction.

Next we verify that (i) holds. In fact,

$$
\begin{aligned}
D^{2} \tilde{\phi}(\tilde{x}) & =D^{2} \tilde{\psi}(\check{\xi}) \\
& =D^{2} \check{u}(\check{\xi})
\end{aligned}
$$

according to (3.15). Since $\check{u}$ is convex, $D^{2} \breve{u}(\breve{\xi}) \geq 0$. Thus, we have condition (i).

Continuing from (3.18) and using (3.15) again, we see that

$$
\begin{aligned}
D^{2} \tilde{\phi}(\tilde{x}) & \geq D^{2} \breve{\psi}(\check{\xi}) \\
& \geq D^{2} \check{\phi}(\check{x})-\frac{\alpha_{0}}{2} I .
\end{aligned}
$$

On the other hand,

$$
\begin{aligned}
\left|D^{2} \check{\phi}(\check{x})-A_{0}\right| & \leq\left|D^{2} \check{\phi}(\check{x})-D^{2} \phi_{j}\left(x_{j}\right)\right|+\left|D^{2} \phi_{j}\left(x_{j}\right)-A_{0}\right| \\
& <\alpha_{1}
\end{aligned}
$$

by (3.10) and (3.12). Therefore, we see from (3.9) that

$$
D^{2} \tilde{\phi}(\tilde{x}) \geq A_{0}-\alpha_{0} I
$$

which is assertion (iii).

Finally, we consider assertion (ii). From (3.16), we have

$$
\begin{aligned}
\left|\tilde{x}-x_{0}\right| & \leq|\tilde{x}-\check{\xi}|+|\check{\xi}-\check{x}|+\left|\check{x}-x_{j}\right|+\left|x_{j}-x_{0}\right| \\
& \leq|\check{\xi}-\check{x}|+\left|\check{x}-x_{j}\right|+\epsilon+\left|x_{j}-x_{0}\right| .
\end{aligned}
$$

Also, since $\check{\xi}$ is a point of twice differentiability with $\tilde{\psi}, \breve{\psi} \in \Xi^{-} \check{u}(\check{\xi})$, we have from definition (3.17)

$$
\begin{aligned}
\left|D \tilde{\phi}(\tilde{x})-p_{0}\right| & =\left|D \tilde{\psi}(\check{\xi})-p_{0}\right| \\
& =\left|D \check{\psi}(\check{\xi})-p_{0}\right| \\
& \leq|D \check{\psi}(\check{\xi})-D \check{\phi}(\check{x})|+\left|D \check{\phi}(\check{x})-D \phi_{j}\left(x_{j}\right)\right|+\left|D \phi_{j}\left(x_{j}\right)-p_{0}\right| .
\end{aligned}
$$

Adding these inequalities and using (3.13), (3.12), and (3.10), we get

$$
\left|\tilde{x}-x_{0}\right|+\left|D \tilde{\phi}(\tilde{x})-p_{0}\right|<\alpha_{0}
$$

which completes the proof of Lemma 3.2.

Forgetting the original sequence $x_{j} \rightarrow x_{0}$ that came from the definition of the closure of the subject, we use Lemma 3.2 in the following form. 
Corollary 3.1.1 There is a sequence $x_{j} \rightarrow x_{0}$ with corresponding $\phi_{j} \in \Xi^{-} u\left(x_{j}\right)$ such that $D^{2} \phi_{j}\left(x_{j}\right) \geq 0$,

$$
\left(u\left(x_{j}\right), D \phi_{j}\left(x_{j}\right)\right) \rightarrow\left(u\left(x_{0}\right), p_{0}\right),
$$

and for any $\alpha_{0}>0$, there is some $j_{0}$ such that

$$
D^{2} \phi_{j}\left(x_{j}\right) \geq A_{0}-\alpha_{0} I
$$

whenever $j \geq j_{0}$.

In light of (3.19), (3.20), the ellipticity of $F$, and (3.8), we may also assume

$$
F\left(x_{j}, u\left(x_{j}\right), D \phi_{j}\left(x_{j}\right), D^{2} \phi_{j}\left(x_{j}\right)\right)>0 .
$$

\section{Preliminary Cases}

If $A_{0}=0$, then since $D \phi_{j}\left(x_{j}\right)=p_{j} \rightarrow p_{0}$ and $\tilde{\phi}_{j}(x)=u\left(x_{j}\right)+p_{j} \cdot\left(x-x_{j}\right)$ is a support plane for $\mathscr{G}$, we see that $\phi_{0}(x)=u\left(x_{0}\right)+p_{0} \cdot\left(x-x_{0}\right)$ is a support plane for $\mathscr{G}$. That is, $\phi_{0} \in \Xi^{-} u\left(x_{0}\right)$. Furthermore,

$$
F\left(x_{0}, u\left(x_{0}\right), D \phi_{0}\left(x_{0}\right), D^{2} \phi_{0}\left(x_{0}\right)\right)=F\left(x_{0}, u\left(x_{0}\right), p_{0}, 0\right)=F\left(x_{0}, u\left(x_{0}\right), p_{0}, A_{0}\right)>0 .
$$

It is not difficult to see that the support hyperplane at the extreme point $X_{0}=$ $\left(x_{0}, u\left(x_{0}\right)\right)$ may be tilted to obtain for any $\alpha>0$ an alternative point $\tilde{X}_{0}=\left(\tilde{x}_{0}, u\left(\tilde{x}_{0}\right)\right)$ and support plane $\tilde{\phi}_{0}(x)=u\left(\tilde{x}_{0}\right)+\tilde{p}_{0} \cdot\left(x-\tilde{x}_{0}\right)$ with

$$
\left\{x: \tilde{\phi}_{0}(x)=u(x)\right\} \subset B_{\alpha}\left(x_{0}\right)
$$

and

$$
\left|\tilde{p}_{0}-p_{0}\right|<\alpha
$$

We prove this assertion under more general circumstances below; see Lemma 3.3. Given $\tilde{x}_{0}$ and $\tilde{\phi}_{0}$, however, we may take $\alpha$ and $\delta$ small enough so that $\psi=\tilde{\phi}_{0}+\delta$ satisfies the assertions of Theorem 3.1. In fact, by continuity, there is some $\tilde{\delta}>0$ such that

$$
\left|u(x)-u\left(x_{0}\right)\right|<\epsilon_{0} / 2 \quad \text { for } x \in B_{\tilde{\delta}}\left(x_{0}\right) .
$$

Also by continuity, we can assume

$$
V=\left\{x: \psi(x)=\tilde{\phi}_{0}(x)+\delta \geq u(x)\right\} \subset B_{2 \alpha}\left(x_{0}\right) \subset B_{\tilde{\delta}}\left(x_{0}\right) \cap B_{\delta_{0}}\left(x_{0}\right) .
$$

It follows that for $x \in V$,

$$
\begin{aligned}
\left|\psi(x)-u\left(x_{0}\right)\right|+\left|D \psi(x)-p_{0}\right| & \leq\left|\tilde{\phi}_{0}(x)-u\left(x_{0}\right)\right|+\delta+\left|\tilde{p}_{0}-p_{0}\right| \\
& <\left|u\left(\tilde{x}_{0}\right)-u\left(x_{0}\right)\right|+\left|\tilde{p}_{0} \cdot\left(x-\tilde{x}_{0}\right)\right|+\delta+\alpha \\
& <\epsilon_{0} / 2+\left|\tilde{p}_{0} \cdot\left(x-x_{0}\right)\right|+\left|\tilde{p}_{0} \cdot\left(x_{0}-\tilde{x}_{0}\right)\right|+\delta+\alpha \\
& <\epsilon_{0} / 2+4\left|\tilde{p}_{0}\right| \alpha+\delta+\alpha \\
& \leq \epsilon_{0} / 2+4\left|\tilde{p}_{0}-p_{0}\right| \alpha+4\left|p_{0}\right| \alpha+\delta+\alpha \\
& <\epsilon_{0} / 2+4 \alpha^{2}+4\left|p_{0}\right| \alpha+\delta+\alpha \\
& <\epsilon_{0},
\end{aligned}
$$


as long as $4 \alpha^{2}+\left(4\left|p_{0}\right|+1\right) \alpha+\delta<\epsilon_{0}$. From this and the fact that $D^{2} \psi \equiv 0=A_{0}$ we obtain $\mathbf{C}_{1}$. Since we may nevertheless take $\delta>0$ and $\psi\left(\tilde{x}_{0}\right)=u\left(\tilde{x}_{0}\right)+\delta$, we see that $\mathbf{B}_{1}$ holds. Finally, $\partial \hat{V}=\{x: \psi(x)=u(x)\}$, so $\mathbf{D}_{1}$ clearly holds.

This discussion serves to rule out the case $A_{0}=0$. The following more general considerations treat the case in which some subsequence of the $\phi_{j}$ satisfies $D^{2} \phi_{j}\left(x_{j}\right)=0$. In this case, we may set $p_{j}=D \phi_{j}\left(x_{j}\right)$ and assume, in light of the ellipticity, that $\phi_{j}(x)=u\left(x_{j}\right)+p_{j} \cdot\left(x-x_{j}\right)$.

Conspicuously absent from the assertions of Corollary 3.1.1 is a statement that $X_{j}=\left(x_{j}, u\left(x_{j}\right)\right)$ is an extreme point. We have, however, that $X_{j} \rightarrow X_{0}$ which is an extreme point. This observation will be used repeatedly below. As an initial application, we prove the tilting lemma mentioned above.

Lemma 3.3 For any $\alpha>0$, if $j$ is large enough, then there is some $\tilde{X}_{j} \equiv\left(\tilde{x}_{j}, u\left(\tilde{x}_{j}\right)\right)$ and support plane $\tilde{\phi}_{j}(x)=u\left(\tilde{x}_{j}\right)+\tilde{p}_{j} \cdot\left(x-\tilde{x}_{j}\right)$ with

$$
\left\{x: \tilde{\phi}_{j}(x)=u(x)\right\} \subset B_{\alpha}\left(x_{j}\right)
$$

and

$$
\left|\tilde{p}_{j}-p_{j}\right|<\alpha
$$

Remark 3.2 If the point at which one wishes to apply this tilting lemma is an extreme point (take $X_{0}$ for example), then a much stronger assertion holds. Namely, there is an arbitrarily close exposed point $\tilde{X}$ with support plane $\tilde{\phi}(x)=u(\tilde{x})+\tilde{p}$. $(x-\tilde{x})$ such that $\left|\tilde{p}-p_{0}\right|$ is small and $\tilde{\phi}(x)<u(x)$ for $x \neq \tilde{x}$. One may use a modification of the reasoning found in the proof of Straszewicz's Theorem in [8] to prove this.

Proof (of Lemma 3.3) Set $F_{j}=\left\{x: \phi_{j}(x)=u\left(x_{j}\right)+p_{j} \cdot\left(x-x_{j}\right)=u(x)\right\}$ for $j=$ $0,1,2, \ldots$. Note that $F_{0}$ is a convex set with $x_{0}$ an extreme point. There is some $p \in \mathbb{S}^{n-1}$ such that

$$
\left\{x \in F_{0}: p \cdot\left(x-x_{0}\right) \geq 0\right\} \subset B_{\alpha / 4}\left(x_{0}\right) .
$$

The existence of $p$ follows from the following more general statement which we prove by induction.

Sublemma 3.1 If $\Pi$ is an $m$-dimensional affine subspace of $\mathbb{R}^{n}$ containing a closed convex subset $F$ with extreme point $x_{0}$, then for any $\alpha>0$, there is some $q \in \mathbb{S}^{n-1}$ and some $\epsilon>0$ such that $x_{0}+q \in \Pi$ and if $p \in \mathbb{S}^{n-1}$ and $|p-q|<\epsilon$, then

$$
\left\{x \in F: p \cdot\left(x-x_{0}\right) \geq 0\right\} \subset B_{\alpha}\left(x_{0}\right) .
$$

Proof If $m=1$, then $F$ is an interval, $\left\{(1-\lambda) x_{1}+\lambda x_{2}: 0 \leq \lambda \leq 1\right\}$, and either $x_{0}=x_{1}$ or $x_{0}=x_{2}$. In the former case, $q=\left(x_{0}-x_{2}\right) /\left|x_{0}-x_{2}\right|$; in the latter $q=$ $\left(x_{0}-x_{1}\right) /\left|x_{0}-x_{1}\right|$.

If $m>1$, then there is some $q_{0}$ such that $x_{0}+q_{0} \in \Pi$ and $q_{0} \cdot\left(x-x_{0}\right) \leq 0$ for every $x \in F$. Note that $\check{\Pi}=\left\{x \in \Pi: q_{0} \cdot\left(x-x_{0}\right)=0\right\}$ is an $(m-1)$-dimensional support plane for $F$ with respect to $\Pi$; see Figure 3 . 


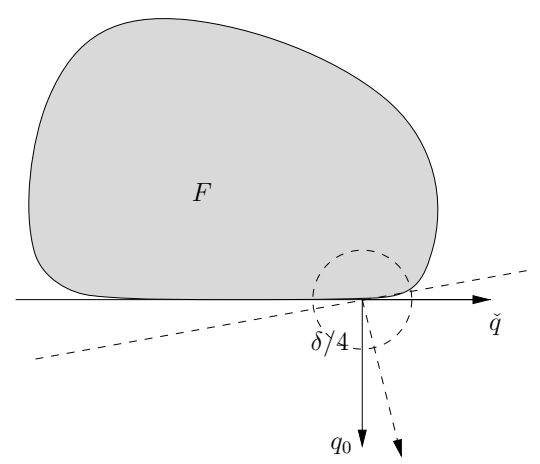

Fig. 3 The neighborhood of an extreme point

Applying the inductive hypothesis to $\check{F}=F \cap \check{\Pi}$, we obtain a vector $\check{q} \in \mathbb{S}^{n-1}$ with $x_{0}+\check{q} \in \check{I}$ and

$$
\left\{x \in \check{F}: \check{q} \cdot\left(x-x_{0}\right) \geq 0\right\} \subset B_{\alpha / 2}\left(x_{0}\right) .
$$

Let $q=q_{0}+a \check{q}$. We claim that when $a$ is small enough, then the assertion of the sublemma holds. If this is not the case, then we obtain sequences $a, \epsilon_{a} \searrow 0$, vectors $p_{\epsilon_{a}} \rightarrow q=q_{0}+a \check{q}$, and points $x_{\epsilon_{a}} \in F \backslash B_{\alpha}\left(x_{0}\right)$ with

$$
p_{\epsilon_{a}} \cdot\left(x_{\epsilon_{a}}-x_{0}\right) \geq 0 \text {. }
$$

On the one hand, since $F$ is convex and $x_{0} \in F$, we may assume that $\left|x_{\epsilon_{a}}-x_{0}\right|=$ $\alpha$. Therefore, taking a subsequence, we may assume that $x_{\epsilon_{a}} \rightarrow z_{a} \in F \cap \partial B_{\alpha}\left(x_{0}\right)$ and from (3.26) we find

$$
q \cdot\left(z_{a}-x_{0}\right) \geq 0
$$

Taking another subsequence, we may also assume $z_{a} \rightarrow z_{0} \in F \cap \partial B_{\alpha}\left(x_{0}\right)$, and from (3.27)

$$
q_{0} \cdot\left(z_{0}-x_{0}\right) \geq 0
$$

It follows then, from the definition of $q_{0}$, that $q_{0} \cdot\left(z_{0}-x_{0}\right)=0$. Therefore, $z_{0} \in$ $\check{\Pi} \cap F=\check{F}$, and by the inductive hypothesis (see (3.25)) we have

$$
\check{q} \cdot\left(z_{0}-x_{0}\right)<0 .
$$

On the other hand, directly from (3.27) we obtain

$$
0 \leq q_{0} \cdot\left(z_{a}-x_{0}\right)+a \check{q} \cdot\left(z_{a}-x_{0}\right),
$$

so that

$$
\check{q} \cdot\left(z_{a}-x_{0}\right) \geq-\frac{1}{a} q_{0} \cdot\left(z_{a}-x_{0}\right) \geq 0 .
$$

It follows that $\check{q} \cdot\left(z_{0}-x_{0}\right) \geq 0$, and this contradicts (3.28). This completes the proof of Sublemma 3.1. 
We now take $p=q$ and return to the proof of Lemma 3.3.

Setting $p_{\epsilon}=p_{0}+\epsilon p$ and

$$
E_{\epsilon}=\left\{x: u\left(x_{0}\right)+p_{\epsilon} \cdot\left(x-x_{0}\right) \geq u(x)\right\}
$$

we claim that for $\epsilon$ small $E_{\epsilon} \subset B_{\alpha / 2}\left(x_{0}\right)$. If this is not the case, then using convexity we obtain a sequence of points $z_{\epsilon} \rightarrow z_{0} \in \partial B_{\alpha / 2}\left(x_{0}\right)$ with

$$
u\left(x_{0}\right)+p_{0} \cdot\left(z_{0}-x_{0}\right) \geq u\left(z_{0}\right) .
$$

That is, $z_{0} \in F_{0}$. Since $\left|z_{0}-x_{0}\right|=\alpha / 2>\alpha / 4$, we conclude from (3.23) that

$$
p \cdot\left(z_{0}-x_{0}\right)<0 \text {. }
$$

On the other hand, since $\phi_{0} \in \Xi^{-} u\left(x_{0}\right)$, we have

$$
\begin{aligned}
\epsilon p \cdot\left(z_{\epsilon}-x_{0}\right) & \geq u\left(z_{\epsilon}\right)-\left[u\left(x_{0}\right)+p_{0} \cdot\left(z_{\epsilon}-x_{0}\right)\right] \\
& =u\left(z_{\epsilon}\right)-\phi_{0}\left(z_{\epsilon}\right) \\
& \geq 0 .
\end{aligned}
$$

From this we see that $p \cdot\left(z_{0}-x_{0}\right) \geq 0$, which contradicts (3.29). Therefore, $E_{\epsilon} \subset$ $B_{\alpha / 2}\left(x_{0}\right)$, and

$$
\delta_{\epsilon}=\max \left\{u\left(x_{0}\right)+p_{\epsilon} \cdot\left(x-x_{0}\right)-u(x): x \in E_{\epsilon}\right\}
$$

is well defined, finite, and attained at some $x_{\epsilon} \in E_{\epsilon} \subset B_{\alpha / 2}\left(x_{0}\right)$. It follows that

$$
\phi_{\epsilon}(x)=u\left(x_{0}\right)+p_{\epsilon} \cdot\left(x-x_{0}\right)-\delta_{\epsilon}=u\left(x_{\epsilon}\right)+p_{\epsilon} \cdot\left(x-x_{\epsilon}\right)
$$

defines a support plane for $\mathscr{G}$ and

$$
F_{\epsilon}=\left\{x: \phi_{\epsilon}(x)=u(x)\right\} \subset B_{\alpha / 2}\left(x_{0}\right) .
$$

Taking $\epsilon$ small enough so that $\left|p_{\epsilon}-p_{0}\right|<\alpha / 2$, then $j$ large enough so that $x_{j} \epsilon$ $B_{\alpha / 2}\left(x_{0}\right)$ and $\left|p_{j}-p_{0}\right|<\alpha / 2$, we may set $\tilde{x}_{j}=x_{\epsilon}, \tilde{p}_{j}=p_{\epsilon}$, and $\tilde{\phi}_{j}=\phi_{\epsilon}$; it is easily verified that these choices fulfil the requirements of Lemma 3.3.

Corollary 3.1.2 Iffor some subsequence $D^{2} \phi_{j}\left(x_{j}\right)=0$ and $\tilde{\phi}_{j}$ are the affine functions given in Lemma 3.3, then $\psi=\tilde{\phi}_{j}+\delta$ satisfies the assertions of Theorem 3.1 when $j$ is large enough and $\delta$ is small enough depending on $j$.

Proof We may take $\alpha_{0}<\min \left\{\epsilon_{0} / 2, \delta_{0} / 4\right\}$ and $j$ large enough in Corollary 3.1.1 so that $\left|x_{j}-x_{0}\right|<\delta_{0} / 2$. By continuity, taking $\delta<\epsilon_{0} / 4$ small enough will ensure by (3.21) that

$$
\hat{V}=\left\{x: \tilde{\phi}_{j}(x)+\delta>u(x)\right\} \subset B_{2 \alpha_{0}}\left(x_{j}\right) \subset B_{\delta_{0}}\left(x_{0}\right) .
$$

It follows that on $\hat{V}$ we have

$$
\left|\tilde{\phi}_{j}(x)+\delta-u(x)\right| \leq \delta<\epsilon_{0} / 4 .
$$

Also by continuity, there is some $\eta>0$ such that $\left|x-x_{0}\right|<\eta$ implies $\mid u(x)-$ $u\left(x_{0}\right) \mid<\epsilon_{0} / 4$. 
As long as $\delta>0$, we know that $\mathbf{B}_{1}$ holds. We may assume $\delta_{0}<\eta$ and $\mid \tilde{p}_{j}-$ $p_{0} \mid<\epsilon_{0} / 2$. Therefore,

$$
\begin{aligned}
\left|\psi(x)-u\left(x_{0}\right)\right|+\left|D \psi(x)-p_{0}\right| & \leq|\psi(x)-u(x)|+\left|u(x)-u\left(x_{0}\right)\right|+\left|\tilde{p}_{j}-p_{0}\right| \\
& <\epsilon_{0},
\end{aligned}
$$

and $\mathbf{C}_{1}$ holds. Finally, $\partial \hat{V}=\{x: \psi(x)=u(x)\}$, so $\mathbf{D}_{1}$ holds.

Another preliminary case is that in which $A_{0}>0$. This implies that for $j$ large, $D^{2} \phi_{j}\left(x_{j}\right)>0$. More generally, we consider the possibility that $D^{2} \phi_{j}\left(x_{j}\right)>0$ is satisfied along some subsequence.

If this occurs, we may take $j$ large enough so that $x_{j} \in B_{\delta_{0} / 2}\left(x_{0}\right)$. Also, from Corollary 3.1.1 we may assume

$$
\begin{gathered}
F\left(x_{j}, u\left(x_{j}\right), D \phi_{j}\left(x_{j}\right), D^{2} \phi_{j}\left(x_{j}\right)\right)>0 \\
\left|\phi_{j}\left(x_{j}\right)-u\left(x_{0}\right)\right|+\left|D \phi_{j}\left(x_{j}\right)-p_{0}\right|<\epsilon_{0} / 2,
\end{gathered}
$$

and

$$
D^{2} \phi_{j}\left(x_{j}\right) \geq A_{0}-\left(\epsilon_{0} / 2\right) I .
$$

Notice that $\phi_{j}$ is strictly convex near $x_{j}$, and for $\epsilon$ small enough $\tilde{\phi}(x)=\phi_{j}(x)-$ $\epsilon\left|x-x_{j}\right|^{2}$ satisfies

$$
\begin{gathered}
\tilde{\phi}(x)<u(x), \quad x \neq x_{j}, \\
\left|\tilde{\phi}\left(x_{j}\right)-u\left(x_{0}\right)\right|+\left|D \tilde{\phi}\left(x_{j}\right)-p_{0}\right|<\epsilon_{0} / 2,
\end{gathered}
$$

and

$$
D^{2} \tilde{\phi}\left(x_{j}\right) \geq A_{0}-\epsilon_{0} I
$$

It follows that for $\delta$ small enough, $\psi(x)=\tilde{\phi}(x)+\delta$ satisfies the assertions of Theorem 3.1 .

This completes our discussion of preliminary cases.

\subsection{Moving to the Origin}

From our discussion so far, we may assume $A_{0}, D^{2} \phi_{j}\left(x_{j}\right) \neq 0$ and $\operatorname{det} A_{0}$, $\operatorname{det} D^{2} \phi_{j}\left(x_{j}\right)=0$ for all $j$. We claim now that by an affine change of variables, we may assume

$$
\left(x_{0}, u\left(x_{0}\right), p_{0}, A_{0}\right)=\left(0,0,0,\left(\begin{array}{ll}
1 & 0 \\
0 & 0
\end{array}\right)\right) .
$$

In fact, there is a rotation matrix $P$ such that

$$
P^{-1} A_{0} P=\left(\begin{array}{cc}
\lambda^{2} & 0 \\
0 & 0
\end{array}\right) .
$$

Set

$$
\tilde{u}(\tilde{x})=u\left(x_{0}+\frac{P}{\lambda} \tilde{x}\right)-u\left(x_{0}\right)-p_{0} \cdot \frac{P}{\lambda} \tilde{x} .
$$


One easily checks that $\tilde{u}$ satisfies the hypotheses of Theorem 3.1 and our desired normalization. Thus, let us assume that for any $\tilde{\delta}_{0}, \tilde{\epsilon}_{0}>0$, we obtain a smooth convex function $\tilde{\psi}: \tilde{V} \rightarrow \mathbb{R}$ satisfying the assertions of Theorem 3.1 (with the appropriate $\sim$ 's inserted).

We may then set

$$
\psi(x)=u\left(x_{0}\right)+p_{0} \cdot\left(x-x_{0}\right)+\tilde{\psi}\left(\lambda P^{-1}\left(x-x_{0}\right)\right)
$$

on $V=P(\tilde{V}) / \lambda+x_{0}$.

Let $\delta_{0}, \epsilon_{0}$ be given in $(0,1)$. Since $\lambda$ is fixed, it is clear that $V \subset B_{\delta_{0}}\left(x_{0}\right)$ if $\tilde{\delta}_{0}$ is small enough. Furthermore, we may take $\tilde{\boldsymbol{\epsilon}}_{0}<1$ small enough so that

$$
\tilde{\boldsymbol{\epsilon}}_{0}<\epsilon_{0} /\left(1+\lambda+\lambda^{2}\right) .
$$

We find that

$$
\begin{aligned}
\psi(x)-u(x) & =u\left(x_{0}\right)-u(x)+p_{0} \cdot\left(x-x_{0}\right)+\tilde{\psi}\left(\lambda P^{-1}\left(x-x_{0}\right)\right) \\
& =\tilde{\psi}(\tilde{x})-\tilde{u}(\tilde{x}),
\end{aligned}
$$

where $\tilde{x}=\lambda P^{-1}\left(x-x_{0}\right)$. Therefore, condition $\mathbf{B}_{1}$ holds. Using the same calculation, we see that for $x=x_{0}+(P / \lambda) \tilde{x} \in \hat{V}$,

$$
\begin{aligned}
|\psi(x)-u(x)|+\left|D \psi(x)-p_{0}\right| & =|\tilde{\psi}(\tilde{x})-\tilde{u}(\tilde{x})|+|\lambda P D \tilde{\psi}(\tilde{x})| \\
& <\tilde{\epsilon}_{0}+\lambda \tilde{\epsilon}_{0} \\
& <\epsilon_{0} .
\end{aligned}
$$

By direct calculation,

$$
\begin{aligned}
D^{2} \psi(x) & =\lambda^{2} P D^{2} \tilde{\psi}(\tilde{x}) P^{-1} \\
& \geq P\left(\begin{array}{cc}
\lambda^{2} & 0 \\
0 & 0
\end{array}\right) P^{-1}-\lambda^{2} \tilde{\epsilon}_{0} I \\
& =A_{0}-\lambda^{2} \tilde{\epsilon}_{0} I \\
& >A_{0}-\epsilon_{0} I .
\end{aligned}
$$

We have established condition $\mathbf{C}_{1}$. Finally, for $x \in \partial \hat{V}$, we have $\tilde{\psi}(\tilde{x}) \leq \tilde{u}(\tilde{x})$. Rearranging this inequality yields $\psi(x) \leq u(x)$ as required by $\mathbf{D}_{1}$.

We now pause to summarize our position in respect to the proof of Theorem 3.1. We have a convex function $u$ in a neighborhood of $0 \in \mathbb{R}^{2}$. The origin is an extreme point for $\mathscr{G}=\operatorname{graph}(u)$, and

$$
\left(p_{0}, A_{0}\right)=\left(0,\left(\begin{array}{ll}
1 & 0 \\
0 & 0
\end{array}\right)\right) \in \operatorname{clos}\left[J^{-} u(0)\right] .
$$

We have a sequence $\phi_{j} \in \Xi^{-} u\left(x_{j}\right)$, from Corollary 3.1.1, with

$$
\begin{gathered}
\left(x_{j}, D \phi_{j}\left(x_{j}\right)\right) \rightarrow(0,0), \\
D^{2} \phi_{j}\left(x_{j}\right) \geq 0,
\end{gathered}
$$


and

$$
D^{2} \phi_{j}\left(x_{j}\right) \geq\left(\begin{array}{ll}
1 & 0 \\
0 & 0
\end{array}\right)-\alpha_{0} I
$$

where $\alpha_{0}>0$ is arbitrary, but $j$ must be large enough. We may also assume $D^{2} \phi_{j}\left(x_{j}\right) \neq 0$ but $\operatorname{det} D^{2} \phi_{j}\left(x_{j}\right)=0$ for all $j$. Notice that since $u(x) \geq u\left(x_{j}\right)+$ $D \phi_{j}\left(x_{j}\right) \cdot\left(x-x_{j}\right)$ and (3.31) holds, we may take a limit as $j \rightarrow \infty$ and conclude $u \geq 0$.

We now proceed to construct the direct modification of Theorem 3.1 for this function in a neighborhood of the origin.

Remark 3.3 The following argument quickly becomes notationally unpleasant. The reader is encouraged to assume, on the first reading, that $\phi_{j} \equiv \phi_{0} \in \Xi^{-} u(0)$.

In light of the last mentioned normalization, for each $j$ there is a rotation

$$
\rho_{j}=\left(\begin{array}{cc}
\cos \theta_{j} & -\sin \theta_{j} \\
\sin \theta_{j} & \cos \theta_{j}
\end{array}\right)
$$

with $\theta_{j} \in[-\pi, \pi]$ such that

$$
\rho_{j}^{-1} D^{2} \phi_{j}\left(x_{j}\right) \rho_{j}=\left(\begin{array}{cc}
\lambda_{j}^{2} & 0 \\
0 & 0
\end{array}\right) .
$$

\section{Lemma 3.4}

$$
\lim _{j \rightarrow \infty} \sin \theta_{j}=0 ; \quad \lim _{j \rightarrow \infty}\left|\cos \theta_{j}\right|=1 ; \quad \underline{\lim _{j \rightarrow \infty}} \lambda_{j}^{2} \geq 1 .
$$

Proof If we assume by way of contradiction that $\lim _{j \rightarrow \infty} \sin \theta_{j} \neq 0$, then by taking a subsequence, we may assume $\theta_{j} \rightarrow \theta_{0}$ with $\sin \theta_{0} \neq 0$. Substituting $D^{2} \phi_{j}\left(x_{j}\right)=$ $\lambda_{j}^{2} \rho_{j} A_{0} \rho_{j}^{-1}$ from (3.32) into (3.20), we obtain

$$
\lambda_{j}^{2}\left(\begin{array}{cc}
\cos ^{2} \theta_{j} & \cos \theta_{j} \sin \theta_{j} \\
\cos \theta_{j} \sin \theta_{j} & \sin ^{2} \theta_{j}
\end{array}\right)-A_{0}+\alpha_{0} I \geq 0 .
$$

Taking the determinant, this implies

$$
\begin{gathered}
\left(\lambda_{j}^{2} \cos ^{2} \theta_{j}-1+\alpha_{0}\right)\left(\lambda_{j}^{2} \sin ^{2} \theta_{j}+\alpha_{0}\right)-\lambda_{j}^{4} \cos ^{2} \theta_{j} \sin ^{2} \theta_{j}= \\
-\lambda_{j}^{2}\left(\sin ^{2} \theta_{j}-\alpha_{0}\right)-\left(1-\alpha_{0}\right) \alpha_{0} \geq 0,
\end{gathered}
$$

which is a contradiction when $\alpha_{0}<\sin ^{2} \theta_{j}$.

Thus, $\sin \theta_{j} \rightarrow 0$ and $\left|\cos \theta_{j}\right| \rightarrow 1$. To see the last assertion of Lemma 3.4, assume that $\lambda_{j}^{2} \rightarrow \lambda_{0}^{2}<1$ in a subsequence. Taking first the limit $j \rightarrow \infty$ in (3.34) and then $\alpha_{0} \rightarrow 0$, we see

$$
\left(\lambda_{0}-1\right) A_{0} \geq 0
$$

which is a contradiction. 
By adjusting our initial change of variable if necessary, and taking a subsequence, we may assume

$$
\lim _{j \rightarrow \infty} \theta_{j}=0
$$

Also, setting

$$
\tilde{\lambda}_{j}^{2}=\min \left\{\lambda_{j}^{2}, 1\right\}
$$

we have from Lemma 3.4

$$
\lim _{j \rightarrow \infty} \tilde{\lambda}_{j}^{2}=1
$$

As mentioned in regard to the tilting lemma (Lemma 3.3), we do not know that $X_{j}=\left(x_{j}, u\left(x_{j}\right)\right)$ is an extreme point but only that $X_{j}$ tends to the extreme point at the origin. An indication of how close $X_{j}$ is to being an extreme point is given by

$$
d_{j}=\max \left\{d: \exists v \in \mathbb{S}^{n} \text { with } X_{j}+\tau v \in \mathscr{G} \text { for all }|\tau| \leq d\right\}
$$

We now make a simple but important observation.

Lemma $3.5 \lim _{j \rightarrow \infty} d_{j}=0$.

Proof If $d_{j} \geq d_{0}>0$ for some subsequence, then (taking a further subsequence) we may assume $v_{j} \rightarrow v_{0} \in \mathbb{S}^{n}$ and $X_{j}+\tau v_{j} \in \mathscr{G}$ for $|\tau| \leq d_{0} / 2$. It follows that $X_{0}+\tau v_{0} \in \mathscr{G}$ for $|\tau| \leq d_{0} / 2$. This contradicts the fact that $X_{0}=(0,0)$ is an extreme point.

For each $j$, we consider

$$
\tilde{u}_{j}(\tilde{x})=u\left(x_{j}+\rho_{j} \tilde{x}\right)-u\left(x_{j}\right)-D \phi_{j}\left(x_{j}\right) \cdot \rho_{j} \tilde{x}
$$

and

$$
\tilde{\phi}_{j}(\tilde{x})=\phi_{j}\left(x_{j}+\rho_{j} \tilde{x}\right)-u\left(x_{j}\right)-D \phi_{j}\left(x_{j}\right) \cdot \rho_{j} \tilde{x} .
$$

One easily verifies $\tilde{\phi}_{j} \in \Xi^{-} \tilde{u}_{j}(0), D \tilde{\phi}_{j}(0)=0$, and

$$
D^{2} \tilde{\boldsymbol{\phi}}_{j}(0)=\left(\begin{array}{cc}
\lambda_{j}^{2} & 0 \\
0 & 0
\end{array}\right) \text {. }
$$

It is also clear that $\tilde{u}_{j}$ is convex, and since $D \tilde{\phi}_{j}(0)=0$, we have

$$
\tilde{u}_{j} \geq 0
$$

Consequently, it is straightforward to check

$$
d_{j}=\max \left\{d: \exists v \in \mathbb{S}^{n-1} \text { with } \tilde{u}_{j}(\tau v)=0 \text { for }|\tau| \leq d\right\}
$$




\subsection{The Main Cases}

At this point it will be convenient to use the old fashioned $(x, y, z)$ notation for points in $\mathbb{R}^{2}$ and $\mathbb{R}^{3}$. As above $B_{r}(0)$ is the open ball of radius $r$. For any domain $D$,

$$
\begin{gathered}
D^{ \pm}=D \cap\{ \pm y \geq 0\} . \\
B_{r_{1}, r_{2}} \equiv \operatorname{int}\left(B_{r_{1}}^{-} \cup B_{r_{2}}^{+}\right) .
\end{gathered}
$$

Since the assertion of Theorem 3.1 is a local one and $x_{j} \rightarrow 0$, we can assume the common domain of definition for the functions $u, \phi_{j}, \tilde{u}_{j}$, and $\tilde{\phi}_{j}$ is a fixed ball $B_{a_{0}}(0)$ with $a_{0}<\delta_{0}$ and that each of the functions $\tilde{u}_{j}$ and $\tilde{\phi}_{j}$ is continuous on the closure of $B_{a_{0}}(0)$.

It will be convenient to suppress some of the `s and $j$ 's, but the reader should keep in mind the change of variables $x=x_{j}+\rho_{j} \tilde{x}$ which will reappear later, n.b., (3.55). Thus, for example $\tilde{\phi}(x, y)$ means $\tilde{\phi}_{j}(\tilde{x}, \tilde{y})$ and it is implicit in results 3.6-3.9 that the assertions only hold for $j$ adequately large.

We will use the existence of the smooth supporting function $\tilde{\phi}$ in various ways, the most basic of which is the following.

Lemma 3.6 For any $\eta>0$, there is an $r=r_{j}>0$ such that

$$
\left(1-\frac{\eta}{2}\right) \tilde{\phi}(x, 0)>\frac{\tilde{\lambda}_{j}^{2}-\eta}{2} x^{2}, \quad 0<|x|<r,
$$

where $\tilde{\lambda}_{j}$ is given in (3.36).

Proof By Taylor's formula,

$$
\left(1-\frac{\eta}{2}\right) \tilde{\phi}(x, y)=\frac{\tilde{\lambda}_{j}^{2}}{2}\left(1-\frac{\eta}{2}\right) x^{2}+\circ\left(x^{2}+y^{2}\right) .
$$

Therefore,

$$
\begin{aligned}
\left(1-\frac{\eta}{2}\right) \tilde{\phi}(x, 0)-\frac{\tilde{\lambda}_{j}^{2}-\eta}{2} x^{2} & =\frac{\eta}{2}\left(1-\frac{\tilde{\lambda}_{j}^{2}}{2}\right) x^{2}+\circ\left(x^{2}\right) \\
& =x^{2}\left(\frac{\eta}{2}\left(1-\frac{\tilde{\lambda}_{j}^{2}}{2}\right)+\frac{\circ\left(x^{2}\right)}{x^{2}}\right) \\
& >0
\end{aligned}
$$

if $0<|x|$ is small enough depending on $j$.

Recalling that $\epsilon_{0}$ is given in the statement of Theorem 3.1, let us fix $\eta<\epsilon_{0} / 16$ and set

$$
\gamma_{j}(x, y)=\gamma_{j}(x)=\frac{\tilde{\lambda}_{j}^{2}-\eta}{2} x^{2}
$$

where $\tilde{\lambda}_{j}$ is given in (3.36). We then have for $j$ large

$$
\left|D^{2} \gamma_{j}-A_{0}\right|<\epsilon_{0} / 8 \text {. }
$$


Under the conditions of Lemma 3.6 we have

$$
\tilde{u}(x, 0) \geq \tilde{\phi}(x, 0) \geq\left(1-\frac{\eta}{2}\right) \tilde{\phi}(x, 0)>\gamma_{j}(x)=\frac{\tilde{\lambda}_{j}^{2}-\eta}{2} x^{2} .
$$

The following elementary observation will also be used several times.

Lemma 3.7 If $(x, y) \rightarrow 0$ and $\tilde{u}(x, y) \leq \gamma_{j}(x, y)+o\left(x^{2}+y^{2}\right)$, then $x / y \rightarrow 0$.

Proof Since $\tilde{\phi}(x, y) \leq \tilde{u}(x, y)$, we have from Taylor's formula,

$$
\frac{\tilde{\lambda}_{j}^{2}}{2} x^{2} \leq \frac{\tilde{\lambda}_{j}^{2}-\eta}{2} x^{2}+\circ\left(x^{2}+y^{2}\right) \text {. }
$$

If $y=0$, we obtain an immediate contradiction, so we may assume that $y>0$. We may also assume without loss of generality that $x \geq 0$. If $\lim \sup (x / y)>0$, then we can assume $y \leq M x$ for some $M>0$. We see then from (3.42)

$$
\frac{\eta}{1+M^{2}} \leq \frac{\eta x^{2}}{x^{2}+y^{2}} \leq \frac{\circ\left(x^{2}+y^{2}\right)}{x^{2}+y^{2}}
$$

which is a contradiction.

Lemma 3.8 The functions $\tilde{u}$ are nonnegative and can be zero only along the $y$ axis.

Proof The nonnegativity of $\tilde{u}$ follows from the fact that $\tilde{u}$ is convex, $\tilde{\phi} \in \Xi^{-} \tilde{u}(0)$, and $D \tilde{\phi}(0)=0$. (Therefore, the plane $z=0$ supports $\tilde{u}$.)

If we assume $\tilde{u}(x, y)=0$ at some point with $x \neq 0$. Then by convexity $\tilde{u}(p)=0$ for $p=\alpha(x, y)$ with $0<\alpha<1$. It follows that the second directional derivative $D_{v v} \tilde{\phi}(0) \leq 0$ in the direction $v=p /|p|$. In light of (3.33), this contradicts (3.37).

Corollary 3.1.3 $\tilde{u}\left(\tau e_{2}\right)=0$ for $|\tau| \leq d_{j}$ where $e_{2}=(0,1)$.

Proof If $d_{j}=0$, then there is nothing to prove. If $d_{j}>0$, then according to (3.39) there is some $v \in \mathbb{S}^{n-1}$ with $\tilde{u}(\tau v)=0$ for $|\tau| \leq d_{j}$. According to Lemma 3.8, all such $\tau v$ must lie along the $y$-axis. Therefore, $v= \pm e_{2}$.

Without loss of generality, we may assume

$$
\tilde{u}_{j}\left(-\tau e_{2}\right)>0 \text { for } \tau>d_{j}
$$

Setting

$$
\delta_{j}=\max \left\{\tau: \tilde{u}_{j}\left(\tau e_{2}\right)=0\right\} \geq d_{j},
$$

we consider

Case 1. For some subsequence, the ratio $d_{j} / \delta_{j} \rightarrow 0$ as $j \rightarrow \infty$. 
This case assumes, naturally, that $\delta_{j}>0$ along the subsequence.

In this case, $\tilde{u}_{j}\left(0, \delta_{j}\right)=0$, and we consider the focal graph based on the curve $\left\{z=\left(\tilde{\lambda}_{j}^{2}-\eta\right) x^{2} / 2, y=0\right\}$ with focal point $\left(0, \delta_{j}, 0\right)$. By this we mean the ruled surface all of whose ruling lines pass through the focal point and the given curve. One finds the focal graph to be the graph of the function $f=f_{j}$ given by

$$
f_{j}(x, y)=\frac{\tilde{\lambda}_{j}^{2}-\eta}{2} \cdot \frac{\delta_{j}}{\delta_{j}-y} \cdot x^{2}
$$

which we may restrict to the rectangular domain $C_{j}=\left[-r_{j}, r_{j}\right] \times\left[-\delta_{j}, \delta_{j}\right)$.

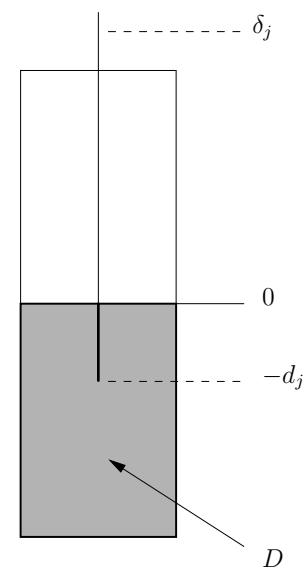

Fig. 4 Rectangular domain along normalized sequence

One easily verifies that $f$ is smooth and convex for $y<\delta_{j}$. In fact,

$$
D f(x, y)=\left(\tilde{\lambda}_{j}^{2}-\eta\right)\left(\frac{\delta_{j}}{\delta_{j}-y}\right)\left(x, \frac{x^{2}}{2\left(\delta_{j}-y\right)}\right) ; D f(0)=0,
$$

and

$$
D^{2} f(x, y)=\left(\tilde{\lambda}_{j}^{2}-\eta\right)\left(\frac{\delta_{j}}{\delta_{j}-y}\right)\left(\begin{array}{cc}
1 & \frac{x}{\delta_{j}-y} \\
\frac{x}{\delta_{j}-y} & \frac{x^{2}}{\left(\delta_{j}-y\right)^{2}}
\end{array}\right) ; D^{2} f(0)=\left(\tilde{\lambda}_{j}^{2}-\eta\right) A_{0} .
$$

Recall that $\delta_{0}, \epsilon_{0}$ are given in the statement of Theorem 3.1, and $\eta$ is fixed with $0<\eta<\epsilon_{0} / 16$. Because $\theta_{j} \rightarrow 0$, we know $\rho_{j} \rightarrow I$, and we may assume

$$
\rho_{j}\left(\begin{array}{ll}
1 & 0 \\
0 & 0
\end{array}\right) \rho_{j}^{-1} \geq\left(\begin{array}{ll}
1 & 0 \\
0 & 0
\end{array}\right)-\frac{\epsilon_{0}}{8} I .
$$

Also, from (3.31) we may assume

$$
\left|x_{j}\right|+\left|u\left(x_{j}\right)\right|+\left|D \phi_{j}\left(x_{j}\right)\right|<\min \left\{\frac{\delta_{0}}{2}, \frac{\epsilon_{0}}{4}, 1\right\} .
$$


We take a smaller domain $C_{a, b}=[-a, a] \times[-b, b]$ with $a<d_{j}, 2 d_{j}<b<3 d_{j}$.

Returning to the expressions (3.45) and (3.46), we observe that for $(x, y) \in$ $C_{a, b}$,

$$
\left|1-\frac{\delta_{j}}{\delta_{j}-y}\right|=\left|\frac{y}{\delta_{j}-y}\right| \leq \frac{3 d_{j}}{\delta_{j}-3 d_{j}}=\frac{3 d_{j} / \delta_{j}}{1-3 d_{j} / \delta_{j}} \rightarrow 0,
$$

and

$$
\left|\frac{x}{\delta_{j}-y}\right| \leq \frac{d_{j}}{\delta_{j}-3 d_{j}} \rightarrow 0
$$

as $j \rightarrow \infty$. Therefore, we may take $j$ large enough so that $C_{a, b} \subseteq B_{\delta_{0} / 2}(0)$, and $f=f_{j}$ satisfies

$$
D^{2} f(x, y) \geq\left(\begin{array}{ll}
1 & 0 \\
0 & 0
\end{array}\right)-\frac{\epsilon_{0}}{8} I
$$

and

$$
|f(x, y)|+|D f(x, y)| \leq \frac{\epsilon_{0}}{8}
$$

for $(x, y) \in C_{a, b}$.

We now make essential use of the convexity of $u$.

\section{Lemma 3.9}

$$
f_{j}(x, y)<\tilde{u}_{j}(x, y) \text { for }(x, y) \in D=\overline{C_{a, b}^{-}} \backslash\left\{(0, y): y \in\left[-d_{j}, 0\right]\right\} .
$$

Proof Let $(x, y) \in D$; see Figure 4. The line segment connecting $(x, y)$ and $\left(0, \delta_{j}\right)$ intersects the $x$-axis in a unique point $(\xi, 0)$ with $\xi=\delta_{j} x /\left(\delta_{j}-y\right)$. Assuming

$$
\tilde{u}(x, y) \leq f_{j}(x, y),
$$

we have from the convexity of $\tilde{u}$ that

$$
\begin{aligned}
\tilde{u}(\xi, 0) & \leq \frac{\delta_{j}}{\delta_{j}-y} \tilde{u}(x, y)-\frac{y}{\delta_{j}-y} \tilde{u}\left(0, \delta_{j}\right) \\
& \leq \frac{\delta_{j}}{\delta_{j}-y} f_{j}(x, y)-\frac{y}{\delta_{j}-y} \tilde{u}\left(0, \delta_{j}\right) \\
& =\frac{\tilde{\lambda}_{j}^{2}-\eta}{2} \xi^{2} .
\end{aligned}
$$

Since $|\xi|<a<r_{j}$, this contradicts (3.41) unless $|\xi|=0$. If $|\xi|=0$, then $y<$ $-d_{j}$, and we find from (3.43) that $f_{j}(x, y)=0<\tilde{u}(x, y)$ which contradicts our assumption (3.52).

In light of (3.51) and (3.50) we have that for $\mu>0$ small

$$
f(x, y)-\mu y<\tilde{u}_{j}(x, y) \quad \text { for }(x, y) \in \partial \overline{C_{a, b}^{-}} \backslash\{0\},
$$

and

$$
|f(x, y)-\mu y|+|D f(x, y)-(0, \mu)|<\frac{\epsilon_{0}}{4} \quad \text { for }(x, y) \in C_{a, b} .
$$


We claim there is some $r^{\prime}<a$ such that

$$
f(x, y)-\mu y<\tilde{u}_{j}(x, y) \quad \text { on }\left(\partial B_{r^{\prime}}(0)\right)^{+} .
$$

Otherwise, we can take $(x, y) \rightarrow 0$ for which $y>0$ and $\tilde{\phi}_{j}(x, y) \leq \tilde{u}_{j}(x, y) \leq$ $f_{j}(x, y)-\mu y$. It is clear that $x \neq 0$, and we may assume without loss of generality that $x>0$. We show, in this case, that $y / x \rightarrow 0$. If $\lim \sup (y / x)>0$, then we can find some $M$ and a subsequence for which $x \leq M y$. Using the first order Taylor formula to express $\tilde{\phi}_{j}$ and $f=f_{j}$, we have

$$
\circ(x+y) \leq-\mu y+\circ(x+y) \text {. }
$$

It follows that $\mu /(M+1) \leq \mu y /(x+y) \leq \circ(x+y) /(x+y)$, which is a contradiction. Thus, $y / x \rightarrow 0$.

On the other hand, the second order Taylor formula shows that $f(x, y)-\mu y<$ $f(x, y)=\left(\tilde{\lambda}_{j}^{2}-\eta\right) x^{2} / 2+\circ\left(x^{2}+y^{2}\right)$. Thus, Lemma 3.7 applies, and we have $x / y \rightarrow 0$. This contradicts our contention that $y / x \rightarrow 0$, and establishes the claim.

The above assertions combine to imply,

$$
\tilde{\psi}(x, y)=f(x, y)-\mu y+\epsilon
$$

satisfies

$$
|\tilde{\psi}|+|D \tilde{\psi}|<\frac{\epsilon_{0}}{2}
$$

and

$$
D^{2} \tilde{\psi} \geq\left(\begin{array}{ll}
1 & 0 \\
0 & 0
\end{array}\right)-\frac{\epsilon_{0}}{8} I
$$

on $\tilde{V}=\operatorname{int}\left(C_{a, b}^{-} \cup B_{r^{\prime}}^{+}\right)$and $\tilde{\psi}_{\left.\right|_{\partial \tilde{V}}}<\tilde{u}_{\left.j\right|_{\partial \tilde{V}}}$, if $\epsilon$ is small enough.

Finally, we take (abandoning for the moment the old fashioned $x, y$-notation)

$$
\psi(x)=u\left(x_{j}\right)+D \phi_{j}\left(x_{j}\right) \cdot\left(x-x_{j}\right)+\tilde{\psi}\left(\rho_{j}^{-1}\left(x-x_{j}\right)\right)
$$

on $V=\rho_{j}(\tilde{V})+x_{j}$. Note that $C_{a, b} \subseteq B_{\delta_{0} / 2}(0)$ and $\left|x_{j}\right|<\delta_{0} / 2$, so $V \subseteq B_{\delta_{0}}(0)$. Also, $\psi\left(x_{j}\right)=u\left(x_{j}\right)+\epsilon$, so $\mathbf{B}_{1}$ holds.

Next, we observe that

$$
D^{2} \psi(x)=\rho_{j} D^{2} \tilde{\psi}(\tilde{x}) \rho_{j}^{-1}=\rho_{j} D^{2} f(\tilde{x}) \rho_{j}^{-1}
$$

where $\tilde{x}=\rho_{j}^{-1}\left(x-x_{j}\right)$. Therefore, referring back to (3.49) and (3.47) we get

$$
D^{2} \psi(x) \geq \rho_{j}\left[\left(\begin{array}{ll}
1 & 0 \\
0 & 0
\end{array}\right)-\frac{\epsilon_{0}}{8} I\right] \rho_{j}^{-1} \geq\left(\begin{array}{ll}
1 & 0 \\
0 & 0
\end{array}\right)-\epsilon_{0} I=A_{0}-\epsilon_{0} I .
$$

Also, in regard to $\mathbf{C}_{1}$, we have from (3.48) and (3.54)

$$
\begin{aligned}
|\psi(x)|+|D \psi(x)| & =\left|u\left(x_{j}\right)+D \phi_{j}\left(x_{j}\right) \cdot\left(x-x_{j}\right)+\tilde{\psi}(\tilde{x})\right|+\left|D \phi_{j}\left(x_{j}\right)-\rho_{j}^{-1} D \tilde{\psi}(\tilde{x})\right| \\
& \leq\left|u\left(x_{j}\right)\right|+2\left|D \phi_{j}\left(x_{j}\right)\right|+|\tilde{\psi}(\tilde{x})|+|D \tilde{\psi}(\tilde{x})| \\
& <\epsilon_{0} .
\end{aligned}
$$


Finally, since $\psi_{\left.\right|_{\partial V}}=u\left(x_{j}\right)+D \phi_{j}\left(x_{j}\right)\left(x-x_{j}\right)_{\left.\right|_{\partial V}}+\tilde{\psi}_{\left.\right|_{\partial \tilde{V}}}<u\left(x_{j}\right)+D \phi_{j}\left(x_{j}\right)(x-$ $\left.x_{j}\right)_{\mid \partial V}+\left.\tilde{u}_{j}\right|_{\partial \tilde{V}}=u_{\left.\right|_{\partial V}}$, we see immediately that $\mathbf{D}_{1}$ holds. This completes Case 1.

The reasoning of Case 1 applies in any circumstances under which the conclusion of Lemma 3.9 holds for any focal graph. More precisely, we have

Corollary 3.1.4 Let $u$ and $\left(p_{0}, A_{0}\right)$ satisfy the hypotheses of Theorem 3.1 and the normalization

$$
\left(p_{0}, A_{0}\right)=\left(0,\left(\begin{array}{ll}
1 & 0 \\
0 & 0
\end{array}\right)\right) .
$$

Assume that for some $x_{j}$ as constructed above, we find

$$
f \leq \tilde{u}_{j} \quad \text { on } C_{a, b}^{-}
$$

where $b>d_{j}$ and $f$ is given by (3.44) or $f=\gamma_{j}$. Assume $f(0, y)<\tilde{u}_{j}(0, y)$ when $y \in\left[-b,-d_{j}\right)$. If $f$ is given by (3.44) rather than $\gamma_{j}$, assume also that $d_{j} / \delta_{j} \rightarrow 0$. Then the conclusion of Theorem 3.1 holds.

Proof Choose $a \leq r_{j}$ if necessary and $\tilde{\delta}_{j} \in\left(\min \left\{\delta_{j} / 2,1\right\}, \delta_{j}\right)$ (where $\delta_{j}=\infty$ if $f=\gamma_{j}$ ). One observes that

$$
\tilde{f}(x, y)=\frac{1-\eta}{2} \cdot \frac{\tilde{\delta}_{j} x^{2}}{\left(\tilde{\delta}_{j}-y\right)} \leq f(x, y) \leq u(x, y)
$$

when $y<0$. The first inequality is strict unless $x=0$. The second inequality is strict when $x=0$ unless $y \in\left[-d_{j}, 0\right)$. Thus, the conclusion of Lemma 3.9 holds with the new focal function $\tilde{f}$ in place of $f$.

One can then follow the reasoning presented above but omit the proof of Lemma 3.9.

Remark 3.4 It follows from Corollary 3.1.4 that we may assume the existence of (a sequence of) points $(\xi, \eta) \rightarrow(0,0)$ satisfying $\tilde{u}_{j}(\xi, \eta)<f(\xi, \eta)$ for any fixed focal graph $f=f_{j}$. Note that Lemma 3.7 applies to these points.

\subsection{The Other Cases}

In all alternatives to Case 1, there is some constant $c>0$ for which

$$
\delta_{j} \leq c d_{j} \rightarrow 0 \quad \text { as } j \rightarrow \infty .
$$

In such situations, we consider the sets

$$
I_{x}=I_{j x}=\left\{y: \gamma_{j}(x)>\tilde{u}_{j}(x, y)\right\} .
$$

Notice that $I_{j 0}=\phi$, and $\gamma_{j}(x)<u(x, 0)$ for $0<|x|<r$, n.b., (3.41). Since for each fixed $x$ (with $0<|x|<r$ ) the function $\gamma_{j}(x)$ is constant and $\tilde{u}(x, y)$ is a convex function of $y$, we see that $I_{x}$ is an interval that does not contain $y=0$. 
Case 2. Some subsequence (in $j$ ) admits $\xi=\xi_{j} \neq 0$ with $I_{x}=\phi$ for $x$ between 0 and $\xi$.

We may assume $\xi>0$. Fix $\eta_{1}>\eta$ such that $\gamma_{1}(x)=\gamma_{1, j}(x)=\left(\tilde{\lambda}_{j}^{2}-\eta_{1}\right) x^{2} / 2$ satisfies

$$
\left|D^{2} \gamma_{1}-A_{0}\right|<\epsilon_{0} / 4
$$

(for $j$ large). Set $m_{j}=\max \left\{\delta_{j}, r_{j}\right\}$. There is some $a<r=r_{j}<\delta_{0}$ and $b \in$ $\left(2 m_{j}, 3 m_{j}\right)$ such that for all $\mu$ and $\epsilon$ adequately small, we have that

$$
\tilde{\psi}(x)=\gamma_{1}(x)+\mu x+\epsilon
$$

satisfies

$$
|\tilde{\psi}|+|D \tilde{\psi}|<\frac{\epsilon_{0}}{2} \quad \text { and } \quad D^{2} \tilde{\psi} \geq A_{0}-\frac{\epsilon_{0}}{4} I
$$

on the rectangle $C_{a, b}$.

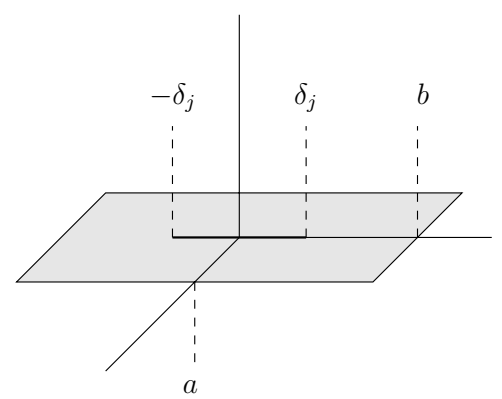

Fig. 5 Rectangular domain for several of the latter cases

Set $V_{1}=\left\{(x, y) \in C_{a, b}: 0 \leq x<\xi\right\}$. Since $\gamma_{1}<\tilde{u}$ on $E=\partial V_{1} \backslash\left\{(0, y):-\delta_{j} \leq\right.$ $\left.y \leq \delta_{j}\right\}$, we see that for $\mu$ small enough

$$
\gamma_{1}(x)+\mu x<\tilde{u}(x, y) \quad \text { for }(x, y) \in E .
$$

On the other hand, for every $\mu>0$ there is some $a^{\prime}<a$ for which

$$
\gamma_{1}(x)+\mu x<0<\tilde{u}(x, y) .
$$

for $(x, y) \in V_{2}=\left\{(x, y) \in C_{a, b}:-a^{\prime} \leq x<0\right\}$.

Setting $\tilde{V}=\operatorname{int}\left(V_{1} \cup V_{2}\right)=\left(a^{\prime}, \min \{a, \xi\}\right) \times(-b, b)$ and

$$
\left\{\begin{array}{l}
V=\rho_{j}\left(\tilde{V}_{j}\right)+x_{j}, \\
\psi(x)=u\left(x_{j}\right)+D \phi_{j}\left(x_{j}\right) \cdot\left(x-x_{j}\right)+\tilde{\psi}\left(\rho_{j}^{-1}\left(x-x_{j}\right)\right),
\end{array}\right.
$$

we see that choosing $j$ large and then $\epsilon$ small,

$$
\begin{gathered}
V \subset B_{\delta_{0}}(0), \\
\psi\left(x_{j}\right)-u\left(x_{j}\right)=\tilde{\psi}(0)=\epsilon>0, \\
\hat{V}=\{x: u(x)<\psi(x)\} \subset \subset V,
\end{gathered}
$$




$$
\begin{aligned}
|\psi(x)|+|D \psi(x)| & \leq\left|u\left(x_{j}\right)\right|+2\left|D \phi_{j}\left(x_{j}\right)\right|+|\tilde{\psi}|+|D \tilde{\psi}| \\
& <\epsilon_{0}
\end{aligned}
$$

and

$$
\begin{aligned}
D^{2} \psi(x) & =\rho_{j} D^{2} \tilde{\psi}(\tilde{x}) \rho_{j}^{-1} \\
& \geq A_{0}-\epsilon_{0} I .
\end{aligned}
$$

This completes the proof of Theorem 3.1 in Case 2.

Case 3. For some subsequence of the $x_{j}$, there are sequences $\xi_{1}=\xi_{1 k}^{j}, \xi_{2}=$ $\xi_{2 k}^{j} \rightarrow 0($ as $k \rightarrow \infty)$ with $\xi_{1} \xi_{2}>0$ and $I_{\xi_{1}}=\phi \neq I_{\xi_{2}}$.

In this case, we may assume $\xi_{1}, \xi_{2} \searrow 0$ (as $k \rightarrow \infty$ ). We take $\tilde{\psi}=\gamma_{j}$ and $\psi$ given by (3.55) as usual. By previous calculations, we may assume

$$
|\psi|+|D \psi|<\epsilon_{0} \quad \text { and } \quad D^{2} \psi \geq A_{0}-\epsilon_{0} I
$$

on all domains $V_{k}=\rho_{j}\left(\tilde{V}_{k}\right)+x_{j}$ where $\tilde{V}_{k}=\left[0, \xi_{1}\right] \times\left[-2 \delta_{j}, 2 \delta_{j}\right]$. We claim that for $k$ large enough, the pair $(\psi, V)=\left(\psi, V_{k}\right)$ just described fulfills the requirements of Theorem 3.1. We may clearly assume $V=V_{k} \subset B_{\delta_{0}}(0)$ and that $\mathbf{C}_{1}$ holds. It remains to verify $\mathbf{B}_{1}$ and $\mathbf{D}_{1}$ (for some fixed large $j$ ).

Let us first show that $\psi_{\left.\right|_{\partial V}} \leq u_{\left.\right|_{\partial V}}$. This is equivalent to showing $\tilde{\psi}_{\left.\right|_{\partial \tilde{V}}} \leq \tilde{u}_{\left.\right|_{\partial \tilde{V}}}$. Note that $\partial \tilde{V}$ is the union of

$$
\begin{aligned}
& A=\left\{(0, y):-2 \delta_{j} \leq y \leq 2 \delta_{j}\right\}, \\
& B=\left\{\left(\xi_{1}, y\right):-2 \delta_{j} \leq y \leq 2 \delta_{j}\right\},
\end{aligned}
$$

and

$$
C_{ \pm}=\left\{\left(x, \pm 2 \delta_{j}\right): 0 \leq x \leq \xi_{1}\right\} .
$$

The desired inequality clearly holds on $A$. Furthermore, since $I_{\xi_{1}}=\phi$, we have that $\tilde{u}\left(\xi_{1}, y\right) \geq \gamma_{j}\left(\xi_{1}\right)=\tilde{\psi}\left(\xi_{1}, y\right)$. This is the desired inequality on $B$. Assume that the inequality fails on $C_{ \pm}=C_{k}$ for every $k$. Then there are points $\xi \rightarrow 0$ with $\tilde{u}\left(\xi, \pm 2 \delta_{j}\right)<\gamma_{j}(\xi)$. Taking the limit as $k \rightarrow 0$, we find that $\tilde{u}\left(0,2 \delta_{j}\right)=0$, which contradicts the definition of $\delta_{j}$. Thus, for $k$ large, assertion $\mathbf{D}_{1}$ of Theorem 3.1 holds.

A slight modification of the reasoning just given for $C_{ \pm}$yields that for $k$ large enough, $\tilde{u}(x, y) \geq \gamma_{j}(x)$ whenever $0 \leq x \leq \xi_{1}$ and $|y| \geq 2 \delta_{j}$. In fact, if $\tilde{u}(\xi, \eta)<$ $\gamma_{j}(\xi)$ for $\xi \rightarrow 0$ and $|\eta| \geq 2 \delta_{j}$, then $\tilde{u}\left(\xi, 2 \delta_{j}\right) \leq \tilde{u}(\xi, \eta) \rightarrow 0$ and we have the same contradiction. Therefore, the fact that $I_{\xi_{2}} \neq \phi$ for some $\xi_{2}<\xi_{1}$ assures us that $\mathbf{B}_{1}$ holds. This completes Case 3.

It follows from the fact that $I_{x}$ is always an interval not containing 0 , that either $I_{x}=I_{x}^{+}=\left\{y \in I_{x}: y \geq 0\right\}$ or $I_{x}=I_{x}^{-}=\left\{y \in I_{x}: y \leq 0\right\}$.

Lemma 3.10 (a) If $\xi_{1} \neq \xi_{2}, \xi_{1} \xi_{2}>0, I_{\xi_{1}}=I_{\xi_{1}}^{+} \neq \phi$, and $I_{\xi_{2}}=I_{\xi_{2}}^{-} \neq \phi$, then there is some $x$ between $\xi_{1}$ and $\xi_{2}$ with $I_{x}=\phi$.

(b) If $I_{x}^{ \pm} \neq \phi$ and $|x|<a$, then $K_{x}^{\mp}=\phi$ where $K_{x}=\left\{y: \gamma_{j}(x) \geq \tilde{u}(x, y)\right\}$. 
Proof We may assume $0<\xi_{2}<\xi_{1}$. It is easy to see that $\left\{\xi: I_{\xi}=I_{\xi}^{+} \neq \phi\right\}$ and $\left\{\xi: I_{\xi}=I_{\xi}^{-} \neq \phi\right\}$ are disjoint open sets. Therefore, $x=\inf \left\{\xi>\xi_{2}: I_{\xi}=I_{\xi}^{-} \neq \phi\right\}$ has $I_{x}=\phi$.

Part (b) follows from the convexity of $u$ and (3.41), n.b., the proof of Lemma 3.9.

Corollary 3.1.5 If none of Cases 1, 2 or 3 hold, then for $j$ large enough, there is some $t_{j}>0$ for which either

Case 4a. $I_{x}=I_{x}^{+} \neq \phi$ for $0<|x|<t_{j}$,

Case 4b. $I_{x}=I_{x}^{-} \neq \phi$ for $0<|x|<t_{j}$,

Case 5a. $I_{x}=I_{x}^{+} \neq \phi$ for $-t_{j}<x<0$ and $I_{x}=I_{x}^{-} \neq \phi$ for $0<x<t_{j}$, or

Case 5b. $I_{x}=I_{x}^{-} \neq \phi$ for $-t_{j}<x<0$ and $I_{x}=I_{x}^{+} \neq \phi$ for $0<x<t_{j}$.

Proof We may consider, at the outset, $j$ large enough so that none of the subsequentially postulated conditions of Cases 1, 2, or 3 apply (at any $j$ ).

Assume there is no $t_{j}$ for which $I_{x}=I_{x}^{+} \neq \phi$ for $-t_{j}<x<0$ and there is no $t_{j}$ for which $I_{x}=I_{x}^{-} \neq \phi$ for $-t_{j}<x<0$.

If there is a sequence $\xi_{1}=\xi_{1 k}^{j} \nearrow 0($ as $k \rightarrow \infty)$ with $I_{\xi_{1}}=I_{\xi_{1}}^{+} \neq \phi$, then either there is a sequence $\xi_{2}=\xi_{2 k}^{j} \nearrow 0$ with $I_{\xi_{2}}=\phi$ or there is a sequence $\xi_{2} \nearrow 0$ with $I_{\xi_{2}}=I_{\xi_{2}}^{-} \neq \phi$. The second possibility reduces to the first by Lemma 3.10, and the first is covered by Case 3 . We conclude that there is no sequence $\xi_{1} \nearrow 0$ with $I_{\xi_{1}}=I_{\xi_{1}}^{+} \neq \phi$.

The same reasoning implies there is no sequence $\xi_{1} \nearrow 0$ with $I_{\xi_{1}}=I_{\xi_{1}}^{-} \neq \phi$. Thus, $I_{x}=\phi$ for all $x$ small enough and negative. But this is covered by Case 2 .

From this contradiction, we conclude that there is some $t_{j}$ for which either $I_{x}=I_{x}^{+} \neq \phi$ for $-t_{j}<x<0$ or $I_{x}=I_{x}^{-} \neq \phi$ for $-t_{j}<x<0$. A similar statement holds for $x>0$, and the remaining cases, listed in the corollary, are simply a listing of the possibilities.

Case 4. We may assume $I_{x}=I_{x}^{+} \neq \phi$ for $0<|x|<t_{j}$.

Since $I_{x}=I_{x}^{+}$, we know that $I_{x}^{-}=\phi$. Therefore, $\gamma_{j}(x) \leq \tilde{u}(x, y)$ for $(x, y) \in$ $C_{t_{j}, b}^{-}$, so Corollary 3.1.4 applies.

Case 5. We may assume $I_{x}=I_{x}^{-} \neq \phi$ for $-t_{j}<x<0$ and $I_{x}=I_{x}^{+} \neq \phi$ for $0<x<t_{j}$.

According to Lemma 3.10(b) $\gamma_{j}(x)<\tilde{u}(x, y)$ for $-t_{j}<x<0$ and $y \geq 0$.

We set

$$
\tilde{\psi}(x, y)=\gamma_{\alpha}(x, y)=\frac{\tilde{\lambda}_{j}^{2}-\eta}{2}(x-\alpha y)^{2} .
$$

We can see easily that $\gamma_{\alpha}$ is smooth and convex. In fact,

$$
\begin{gathered}
\gamma_{\alpha}(0)=0 . \\
D \gamma_{\alpha}(x, y)=\left(\tilde{\lambda}_{j}^{2}-\eta\right)(x-\alpha y)(1,-\alpha) ; D \gamma_{\alpha}(0)=0 .
\end{gathered}
$$


and

$$
D^{2} \gamma_{\alpha}(x, y)=\left(\tilde{\lambda}_{j}^{2}-\eta\right)\left(\begin{array}{cc}
1 & -\alpha \\
-\alpha & \alpha^{2}
\end{array}\right) .
$$

Moreover, $\gamma_{\alpha}$ converges uniformly and smoothly on compact subsets to $\gamma_{j}$ as $\alpha \rightarrow 0$.

We take $a<r_{j}<\delta_{0}$ and $2 \delta_{j}<b<3 \delta_{j}$ so that

$$
\left|\gamma_{j}(x, y)\right|+\left|D \gamma_{j}(x, y)\right|<\frac{\epsilon_{0}}{4} \quad \text { and } \quad D^{2} \gamma_{j}(x, y)>A_{0}-\frac{\epsilon_{0}}{4} I
$$

whenever $(x, y) \in C_{a, b}$. For all $\alpha$ small enough, we have

$$
\left|\gamma_{\alpha}(x, y)\right|+\left|D \gamma_{\alpha}(x, y)\right|<\frac{\epsilon_{0}}{2} \quad \text { and } \quad D^{2} \gamma_{\alpha}(x, y)>A_{0}-\frac{\epsilon_{0}}{2} I
$$

when $(x, y) \in C_{a, b}$.

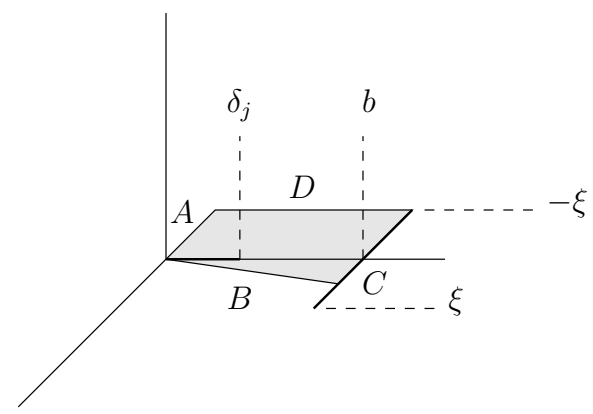

Fig. 6 Modified domain for Case 5

Setting this aside for a moment, we take a sequence $\xi=\xi_{k}^{j} \searrow 0$ and set

$$
\begin{aligned}
& A=\{(x, 0):-\xi \leq x \leq 0\}, \\
& B=\{y(\alpha, 1): 0 \leq y \leq b\}, \\
& C=\{(x, b):-\xi \leq x \leq \xi\}, \\
& D=\{(-\xi, y): 0 \leq y \leq b\} .
\end{aligned}
$$

It is clear that $\psi=\gamma_{\alpha} \leq \tilde{u}$ on $A \cup B$. (Note that $\gamma_{\alpha}=0$ on $B$.)

Moreover according to Lemma 3.10, $\gamma_{j}<\tilde{u}$ for $-\xi<x<0$ and $y \geq 0$. This includes $D$ in particular. Also, if $\xi$ is small enough, then $\gamma_{j}<\tilde{u}$ on $C=C_{k}$. To see this, assume there are points $\left(\xi_{1}, b\right)$ with $-\xi \leq \xi_{1} \leq \xi$ and $\tilde{u}(\xi, b) \leq \gamma_{j}(\xi, b)$. Since $\xi \rightarrow 0$, we can take a limit and find $u(0, b)=0$ which is a contradiction. Thus, we can fix $\xi$ small enough that

$$
\gamma_{j}<u \quad \text { on } C \cup D .
$$


Since $C \cup D$ is a compact set and $\gamma_{\alpha} \rightarrow \gamma_{j}$, we find that for $\alpha$ small enough, in addition to (3.60) we have

$$
\gamma_{\alpha}<\tilde{u} \quad \text { on } C \cup D \text {. }
$$

We now choose $\tilde{V}=\tilde{V}_{\alpha}=\left\{(x, y) \in C_{a, b}^{+}:-\xi<x<\alpha y\right\}$. Choosing $\alpha$ smaller once more if necessary, we see that $\partial \tilde{V}_{\alpha} \subset A \cup B \cup C \cup D$, so that $\tilde{\psi}_{\left.\right|_{\partial \tilde{V}}} \leq \tilde{u}_{\left.\right|_{\partial \tilde{V}}}$. With the usual definitions for $V$ and $\psi$ given in (3.58), we see as usual that $\mathbf{D}_{1}$ holds. Also, $\mathbf{C}_{1}$ follows from (3.60) and the usual estimates. It only remains to find a point in $\hat{V}$ or equivalently a point in $(x, y) \in \tilde{V}$ for which $\tilde{u}(x, y)<\tilde{\psi}(x, y)=$ $\gamma_{\alpha}(x, y)$.

Consider any sequence $\xi_{1}=\xi_{1 k}^{j} \searrow 0$. Since $I_{\xi_{1}}=I_{\xi_{1}}^{+} \neq \phi$, there exist $\eta_{1}>0$ for which

$$
\tilde{u}\left(\xi_{1}, \eta_{1}\right)<\gamma_{j}\left(\xi_{1}\right)
$$

We first observe that $\eta_{1} \rightarrow 0$, for if not, we can take a subsequence for which $\eta_{1} \rightarrow \eta_{0}>0$, and by continuity obtain $\tilde{u}\left(0, \eta_{0}\right)=0$ (a contradiction). Therefore, Lemma 3.7 applies, and we see that $\xi_{1} / \eta_{1} \rightarrow 0$. It follows that there are points $\left(\xi_{1}, \eta_{1}\right) \in \tilde{V}$ for which $\tilde{u}\left(\xi_{1}, \eta_{1}\right)<\gamma_{j}\left(\xi_{1}\right)$ and

$$
0<\xi_{1} \leq \frac{\alpha}{2} \eta_{1}<\alpha \eta_{1} \text {. }
$$

We observe,

$$
\begin{aligned}
\tilde{\psi}\left(\xi_{1}, \eta_{1}\right) & =\gamma_{\alpha}\left(\xi_{1}, \eta_{1}\right) \\
& =\frac{\tilde{\lambda}_{j}^{2}-\eta}{2}\left(\xi_{1}-\alpha \eta_{1}\right)^{2} \\
& >\frac{\tilde{\lambda}_{j}^{2}-\eta}{2} \xi_{1}^{2} \\
& >u\left(\xi_{1}, \eta_{1}\right) .
\end{aligned}
$$

Thus, $x_{j}+\rho_{j}\left(\xi_{1}, \eta_{1}\right) \in \hat{V} \neq \phi$.

\section{References}

1. Alvarez, O., Lasry, J.M., Lions, P.L.: Convex viscosity solutionsand state constraints. J. Math. Pures Appl. 9, no. 3, 265-288 (1997)

2. Andrews, B., Feldman, M.: Nonlocal geometric expansion of convex planar curves. J. Differential Equations 182, no. 2, 298-343 (2002)

3. Crandall, M., Ishii, H., Lions, P.L.: User's guide to viscosity solutions of second order partial differential equations. Bull. Amer. Math. Soc. 27, no. 1, 1-67 (1992)

4. Gilbarg, D., Trudinger, N.S.: Elliptic Partial Differential Equations of Second Order. Second edition. Springer-Verlag, Berlin (1983)

5. Ishii, H.: Perron's method for Hamilton-Jacobi equations. Duke Math. J. 55, no. 2, 369-384 (1987)

6. Jensen, R.: The maximum principle for viscosity solutions of fully nonlinear second order partial differential equations. Arch. Rat. Mech. Anal. 101, 1-27 (1988)

7. Jensen, R.: Uniquess criteria for viscosity solutions of fully nonlinear elliptic partial differential equations. Indiana Univ. Math. J. 38, 629-667 (1989)

8. Schneider, R.: Convex Bodies: The Brunn-Monkowski Theory. Encyclopedia of mathematics and its applications, vol. 44. Cambridge University Press, Cambridge (1993)

9. Soner, H.M.: Motion of a set by the curvature of its boundary. J. Differential Equations 101, no. 2, 313-372 (1993) 